\title{
OPEN Biomarkers of cardiometabolic complications in survivors of childhood acute lymphoblastic leukemia
}

\author{
Sophia Morel ${ }^{1,5}$, Pauline Léveillé ${ }^{1,2}$, Mariia Samoilenko ${ }^{1,3}$, Anita Franco ${ }^{1}$, Jade England ${ }^{1,4}$, \\ Nicolas Malaquin ${ }^{6}$, Véronique Tu ${ }^{6}$, Guillaume B. Cardin ${ }^{6}$, Simon Drouin ${ }^{1}$, Francis Rodier ${ }^{6,7}$, \\ Sarah Lippé ${ }^{1,2}$, Maja Krajinovic ${ }^{1,4}$, Caroline Laverdière ${ }^{4}$, Daniel Sinnett ${ }^{1,4}$, \\ Geneviève Lefebvre ${ }^{3}$, Emile Levy ${ }^{1,5}$ \& Valérie Marcil ${ }^{1,5 \bowtie}$
}

Survivors of childhood acute lymphoblastic leukemia (CALL) are at higher risk of developing cardiometabolic complications. We aimed at exploring the associations between biomarkers of inflammation, oxidative stress, endothelial function, endotoxemia and cardiometabolic risk factors. We conducted a cross-sectional analysis in 246 cALL survivors (mean age, $22.1 \pm 6.3$ years; mean time since diagnosis, $15.5 \pm 5.2$ years) and evaluated the associations using a series of logistic regressions. Using structural equation models, we also tested if the relationship between endotoxemia and cardiometabolic complications was mediated by the latent (unobserved) variable inflammation inferred from the observed biomarkers CRP, TNF- $\alpha$ and IL-6. High leptin-adiponectin ratio was associated with obesity [adjusted $\mathrm{OR}=15.7 ; 95 \% \mathrm{Cl}(6.2-39.7)]$, insulin resistance [20.6 (5.2-82.1)] and the metabolic syndrome [11.2 (2.6-48.7)]. Higher levels of plasminogen activator inhibitor-1 and tumor necrosis factor- $\alpha$ were associated with obesity [3.37 (1.6-7.1) and 2.34 (1.3-4.2), respectively] whereas high C-reactive protein levels were associated with insulin resistance [3.3 (1.6-6.8)], dyslipidemia [2.6 (1.4-4.9)] and MetS [6.5 (2.4-17.9)]. Our analyses provided evidence for a directional relationship between lipopolysaccharide binding protein, related to metabolic endotoxemia, inflammation and cardiometabolic outcomes. Identification of biomarkers and biological mechanisms could open new avenues for prevention strategies to minimize the long-term sequelae, improve follow-up and optimize the quality of life of this high-risk population.

Survivors of childhood acute lymphoblastic leukemia (cALL) are at increased risk of long-term cardiometabolic complications including obesity, insulin resistance, dyslipidemia and hypertension ${ }^{1-4}$. While the precise etiology of these long-term complications is not fully understood, cranial radiation therapy (CRT) and chemotherapy have been proposed as contributing factors ${ }^{3,5}$. Possible underlying mechanisms include oxidative stress, chronic inflammation, adipose tissue dysfunction, endocrine disorders and accelerated cellular aging (reviewed in ${ }^{6}$ ). Identifying biomarkers related to long-term treatment outcomes could improve our knowledge on the biological factors influencing them and help predicting these morbidities in individuals and/or subgroups of patients.

In young adult survivors of cALL, the presence of systemic inflammation and increased activation of the immune system have been demonstrated ${ }^{7-9}$. In parallel, studies found high levels of adipokines and pro-inflammatory cytokines during ${ }^{10-13}$ and after ${ }^{14,15}$ chemotherapy, up to 5 years after the initiation of treatment ${ }^{16}$. Exposure to CRT can affect several metabolic pathways and thus promoting weight gain, insulin resistance and hormonal deficiencies ${ }^{17}$. Excess adipose tissue accompanying obesity promotes the synthesis and release of adipokines along with the development of a systemic inflammatory state, which may affect insulin sensitivity and vascular

\footnotetext{
${ }^{1}$ Research Centre of Sainte-Justine University Health Center, Université de Montréal, 3175 Côte Ste-Catherine room 4.17.006, Montreal, OC H3T 1C5, Canada. '2Department of Psychology, Université de Montréal, Montreal, OC H3T 1C5, Canada. ${ }^{3}$ Department of Mathematics, Université du Québec à Montréal, Montreal, OC H3C 3P8, Canada. ${ }^{4}$ Department of Pediatrics, Université de Montréal, Montreal, OC H3T 1C5, Canada. ${ }^{5}$ Department of Nutrition, Université de Montréal, Montreal, OC H3T 1C5, Canada. ${ }^{6} \mathrm{CRCHUM}$ and Institut du Cancer de Montréal, Montreal, OC H2X 0A9, Canada. 'Department of Radiology, Radio-Oncology, and Nuclear Medicine, Université de Montréal, Montreal, OC H3T 1C5, Canada. ${ }^{\circledR}$ email: valerie.marcil@umontreal.ca
} 
function ${ }^{18}$. Consequently, some adipokines including leptin and adiponectin have been proposed as biomarkers of metabolic disturbances in populations of childhood cancer survivors ${ }^{19-22}$, but all studies stressed the need for validating these findings in additional cohorts.

Endothelial dysfunction was reported in long-term survivors of $\mathrm{CALL}^{23}$, which seemingly contributes to their greater risk for cardiovascular disease development ${ }^{24-28}$. The infiltration and retention of low-density lipoprotein (LDL) in the arterial intima initiate an inflammatory endothelial process leading to atherosclerotic plaque formation. Modification of LDL through oxidation causes endothelial cells to express leucocyte adhesion molecules. In cALL survivors, circulatory levels of the adhesion molecules intracellular adhesion molecule 1 (ICAM-1) ${ }^{29}$, vascular adhesion molecule-1 (VCAM-1) $)^{30}$ and E-selectin ${ }^{31}$ were found elevated, although their relationship with cardiometabolic outcomes was not assessed.

Many chemotherapeutic agents induce oxidative stress and trigger an inflammatory response ${ }^{7-9,32-34}$. Oxidative stress is caused by the imbalance between the generation of free radicals and antioxidant defenses. If reactive oxygen species are not scavenged, they may conduct to widespread lipid, protein and DNA damage. Systemic inflammation also increases the susceptibility for oxidative modifications by endothelial and smooth muscle cells to LDL. To control the flux of reactive oxygen species, aerobic cells possess endogenous antioxidant enzymes that include superoxide dismutases (SOD) and glutathione peroxidases (GPx). Although several studies reported changes in antioxidant enzyme activities in serum leukocytes and red blood cells of leukemia patients during pathogenesis of leukemia and/or treatment (reviewed $\mathrm{in}^{35}$ ), findings are discordant and studies have not addressed these aspects in cancer survivors. Besides, mitochondrion is implicated in a variety of cellular functions including cell signaling, metabolism, cell death, aging, and cancer ${ }^{36}$. Variations in mitochondrial DNA $(\mathrm{mtDNA})^{37,38}$ and in the regulation of mitochondrial proteins related to inflammation and to antioxidant activity have been identified in cALL survivors ${ }^{39}$, but no prior study has examined the relationship between mtDNA and cardiometabolic complications in this particular group of patients.

Endotoxins derived from intestinal bacteria are among the factors triggering a peripheral inflammatory state. Accordingly, the gut microbiota is recognized as a contributor to the systemic inflammation ${ }^{40}$ and oxidative stress $^{41}$, which can remotely affect peripheral organs implicated in the development of obesity, insulin resistance and atherosclerosis ${ }^{42,43}$. Release of bacterial lipopolysaccharides (LPS), produced by Gram-negative bacteria, contribute to chronic ${ }^{44}$ and adipose tissue ${ }^{45}$ inflammation. LPS-binding protein (LBP) is produced mainly by the liver and helps mediate the LPS-induced inflammatory response ${ }^{46}$. Not only gut microbiota influence blood levels of LPS, but intestinal counts of Gram-negative bacteria was found correlated to LBP ${ }^{47}$. It is possible that endotoxemia, caused by changes in the intestinal microbiota during cALL treatment, triggers a pro-oxidative and pro-inflammatory state leading to the development of cardiometabolic complications.

There is a need to validate the utility of biomarker testing in order to characterize subgroups of patients most susceptible of developing late cardiometabolic complications. Therefore, our first objective was to explore the associations between blood biomarkers of oxidative stress, inflammation, endothelial function and endotoxemia and cardiometabolic risk factors in CALL survivors. Our second objective was to test, using structural equation models, if the relationship between endotoxemia and cardiometabolic complications was mediated by the latent (unobserved) variable inflammation inferred from the observed biomarkers CRP, TNF- $\alpha$ and IL-6.

\section{Results}

Cohort characteristics. Relevant demographic and treatment characteristics are outlined in Table 1. Median age at interview was 21.8 years, ranging from 8.5 to 41.0 years, and median time elapsed since diagnosis was 15.2 years. A total of 146 participants (59.4\%) had received CRT (dose range: 10-19.8 Gy, with $47 \%$ of survivors having received $\geq 18 \mathrm{~Gy}$ ). Dyslipidemia was the most prevalent cardiometabolic complication (41.5\%), followed by obesity $(32.5 \%)$, insulin resistance $(17.1 \%)$ and pre-hypertention (pre-HTN) or hypertension (HTN) (12.2\%). Among the types of anomalies that define dyslipidemia, $12.2 \%$ had elevated triglycerides, $17.5 \%$ high LDL-cholesterol (LDL-C) and 23.2\% low high-density lipoprotein-cholesterol (HDL-C) levels. Additionally, metabolic syndrome (MetS) affected a total of 22 participants $(9.0 \%)$.

Associations between biomarkers and cardiometabolic complications. In preliminary analyses, all biomarkers were tested in one third of participants and associations with cardiometabolic outcomes were assessed. Testing was carried out in additional participants when associations were significant or close to significance. Depending on the biomarkers, data of 79 to 244 participants were analyzed (Table 2).

The associations between biomarkers of inflammation and cardiometabolic complications are outlined in Table 3. Adjusted models revealed that higher adiponectin levels were protective of obesity $(\mathrm{OR}=0.18 ; 95 \% \mathrm{CI}$ $0.09-0.38)$, insulin resistance $(\mathrm{OR}=0.17 ; 95 \% \mathrm{CI} 0.07-0.45)$ and dyslipidemia $(\mathrm{OR}=0.34 ; 95 \% \mathrm{CI} 0.18-0.66)$. Furthermore, participants with higher adiponectin levels were at lower risk of having MetS $(\mathrm{OR}=0.07 ; 95 \%$ CI 0.01-0.38). Conversely, the risk of obesity and insulin resistance was associated with greater leptin levels $(\mathrm{OR}=9.57 ; 95 \%$ CI 3.41-26.86 and $\mathrm{OR}=13.17$; 95\% CI 3.68-47.10, respectively). The previous results are reflected in leptin-adiponectin ratio deleterious associations. The latter was associated with the risk of obesity $(\mathrm{OR}=15.69 ; 95 \% \mathrm{CI}$ 6.20-39.67), insulin resistance $(\mathrm{OR}=20.60 ; 95 \% \mathrm{CI} 5.17-82.14)$ and $\mathrm{MetS}(\mathrm{OR}=11.20 ; 95 \%$ CI 2.58-48.69). High PAI-1 levels were associated with the risk of having obesity (OR=3.37; 95\% CI 1.61-7.05). Higher CRP levels ( $>3 \mathrm{mg} / \mathrm{L}$ vs. $\leq 3 \mathrm{mg} / \mathrm{L}$ ) were associated with insulin resistance $(\mathrm{OR}=3.27 ; 95 \% \mathrm{CI} 1.58-6.79)$, dyslipidemia $(\mathrm{OR}=2.61 ; 95 \% \mathrm{CI} 1.40-4.86)$ and MetS $(\mathrm{OR}=6.52 ; 95 \% \mathrm{CI} 2.28-17.85)$. Participants with higher TNF- $\alpha$ levels were at increased risk of having obesity $(\mathrm{OR}=2.34 ; 95 \% \mathrm{CI} 1.31-4.18)$. Higher visfatin levels were protective of insulin resistance $(\mathrm{OR}=0.27 ; 95 \% \mathrm{CI} 0.11-0.66)$.

Among the biomarkers of oxidative stress measured, only oxidized-LDL (Ox-LDL) concentrations were associated with dyslipidemia (OR=7.90; 95\% CI 3.80-16.4). After correction for multiple testing, GSH, GPx, 


\begin{tabular}{|c|c|}
\hline & Total $(n=246)$ \\
\hline \multicolumn{2}{|l|}{ Sex N (\%) } \\
\hline Male & $121(49.2)$ \\
\hline Female & $125(50.8)$ \\
\hline \multicolumn{2}{|l|}{ Age at interview, years } \\
\hline Mean (SD) & $22.1(6.3)$ \\
\hline Median (range) & $21.8(8.5-41.0)$ \\
\hline \multicolumn{2}{|c|}{ Age at cancer diagnosis, years } \\
\hline Mean (SD) & $6.6(4.6)$ \\
\hline Median (range) & $4.8(0.9-18.0)$ \\
\hline \multicolumn{2}{|c|}{ Time since diagnosis, years } \\
\hline Mean (SD) & $15.5(5.2)$ \\
\hline Median (range) & $15.2(5.4-28.2)$ \\
\hline CRT exposure $\mathrm{N}(\%)$ & $146(59.4)$ \\
\hline \multicolumn{2}{|c|}{ Cardiometabolic complication N (\%) } \\
\hline Obesity & $80(32.5)$ \\
\hline Insulin resistance & $42(17.1)$ \\
\hline Pre-HTN/HTN & $30(12.2)$ \\
\hline Dyslipidemia & $102(41.5)$ \\
\hline Low HDL-C & $57(23.2)$ \\
\hline High LDL-C & $43(17.5)$ \\
\hline High TG & $30(12.2)$ \\
\hline Metabolic syndrome & $22(9.0)$ \\
\hline
\end{tabular}

Table 1. Demographic and clinical characteristics of participants. Metabolic syndrome was defined according to the International Diabetes Federation. CRT cranial radiotherapy; HDL-C high-density lipoproteincholesterol; HTN hypertension; LDL-C low-density lipoprotein-cholesterol; SD standard deviation; TG triglycerides.

SOD and protein carbonyl levels were not associated with any cardiometabolic complication (Table 4). LBP, a biomarker of endotoxemia, was associated with obesity $(\mathrm{OR}=2.03$; 95\% CI 1.13-3.65) and dyslipidemia $(\mathrm{OR}=1.92$; 95\% CI 1.09-3.37) (Table 5). Levels of ICAM-1 were associated with obesity $(\mathrm{OR}=2.15 ; 95 \% \mathrm{CI} 1.21-3.82)$ and MetS (OR=3.60; 95\% CI 1.32-9.84) (Table 6).

Among the disturbances that define dyslipidemia, only an association with low HDL-C was observed. High LBP levels were associated with low HDL-C (OR=3.26; 95\% CI 1.37-7.73) (Supplementary Table S2). No other association was found when the associations between the biomarkers and the risk of having high triglycerides, high LDL-C, and low HDL-C were analyzed individually (Supplementary Tables S3 to S5).

Structural equation models to assess LBP-inflammation-cardiometabolic complication relationships. For two SEM considered (see Figs. 1 and 2), estimated factor loadings of CRP, TNF- $\alpha$ and IL-6 for the latent variable inflammation, as well as the path coefficients reflecting the relationships between LBP, inflammation and the cardiometabolic outcomes are presented in Tables 7 and 8 . As indicated by the goodness-of-fit measures, the models hypothesized generally appeared to fit well the data ${ }^{48,49}$. Estimated path coefficients representing relationships between LBP, inflammation and cardiometabolic outcomes indicated expected significant directional relationships with the exception of hypertension (Table 7). The standardized regression coefficients between LBP, inflammation and MetS were also statistically significant (Table 8). Of note, the covariables age at interview and sex were associated with dyslipidemia and hypertension respectively (Table 7). The covariable exposition to CRT was not associated with cardiometabolic outcomes (Tables 7 and 8).

\section{Discussion}

We found associations between several inflammatory biomarkers and obesity, dyslipidemia and insulin resistance in a cohort of adolescent and young adult survivors of cALL. Our analyses support the hypothesis of a directional relationship between LBP, a biomarker of endotoxemia, inflammation and cardiometabolic outcomes. These results are in agreement with the hypothesis that, in cALL survivors, endotoxemia triggers a pro-inflammatory state that is associated with the development of cardiometabolic complications.

We found that high TNF- $\alpha$ levels were associated with the risk of obesity. TNF- $\alpha$ contributes to metabolic dysregulation by impairing lipid storage and oxidative capacity of adipose tissues ${ }^{50}$. TNF- $\alpha$ can impact whole body insulin sensitivity through increased free fatty acids and altered adipokine production ${ }^{50}$. Our finding corroborates a large body of literature showing a strong correlation between TNF- $\alpha$ plasma levels and obesity [reviewed in ${ }^{51}$, as well as its relationship with BMI in obese children ${ }^{52,53}$ and adolescents $^{54}$.

Our analyses also revealed that high CRP levels are associated with the risk of insulin resistance, dyslipidemia and MetS. CRP is an acute-phase protein secreted by the liver and the adipose tissue in response to 


\begin{tabular}{|c|c|c|}
\hline Biomarker & Median & Range \\
\hline \multicolumn{3}{|l|}{ Biomarkers of inflammation } \\
\hline Adiponectin $(\mathrm{ng} / \mathrm{ml}) \mathrm{n}=176$ & 14.8 & $1.02-43.9$ \\
\hline Leptin $(\mu \mathrm{g} / \mathrm{ml}) \mathrm{n}=157$ & 11.3 & $0.43-50.0$ \\
\hline Resistin $(\mu \mathrm{g} / \mathrm{ml}) \mathrm{n}=87$ & 4.88 & $1.03-13.3$ \\
\hline Visfatin $(\mathrm{pg} / \mathrm{ml}) \mathrm{n}=161$ & 81.7 & $2.39-539$ \\
\hline $\mathrm{IL}-6(\mathrm{pg} / \mathrm{ml}) \mathrm{n}=237$ & 0.36 & $0.01-9.01$ \\
\hline $\mathrm{TNF}-\alpha(\mathrm{pg} / \mathrm{ml}) \mathrm{n}=244$ & 2.06 & $0.83-55.2$ \\
\hline PAI-1 (ng/ml) n= 145 & 17.9 & $0.54-155$ \\
\hline $\mathrm{CRP}(\mathrm{mg} / \mathrm{l}) \mathrm{n}=238$ & 1.00 & $0.00-22.0$ \\
\hline \multicolumn{3}{|l|}{ Biomarkers of oxidative stress } \\
\hline $\mathrm{SOD}(\mathrm{U} / \mathrm{ml}) \mathrm{n}=156$ & 105 & $55.8-167$ \\
\hline $\mathrm{GPx}(\mathrm{nmol} / \mathrm{min} / \mathrm{ml}) \mathrm{n}=244$ & 632 & $139-1414$ \\
\hline GSH (nmol/mg proteins) $\mathrm{n}=240$ & 42.3 & $18.6-77.1$ \\
\hline Ox-LDL $(U / \mathrm{l}) \mathrm{n}=169$ & 53.2 & $19.3-101$ \\
\hline Protein carbonyls $(\mathrm{nmol} / \mathrm{mg}) \mathrm{n}=87$ & 0.59 & $0.11-1.90$ \\
\hline mtDNA (relative gene expression) $n=227$ & 4.68 & $2.15-7.56$ \\
\hline \multicolumn{3}{|l|}{ Biomarkers of endotoxemia } \\
\hline LPS $(\mathrm{ng} / \mathrm{ml}) \mathrm{n}=125$ & 12.5 & $2.20-26.6$ \\
\hline $\mathrm{LBP}(\mu \mathrm{g} / \mathrm{ml}) \mathrm{n}=244$ & 18.8 & $4.25-48.3$ \\
\hline \multicolumn{3}{|l|}{ Biomarkers of endothelial function } \\
\hline ICAM-1 (ng/ml) n = 244 & 537 & $284-1343$ \\
\hline VCAM-1 (ng/ml) n = 244 & 695 & $365-1573$ \\
\hline E-Selectin $(\mathrm{ng} / \mathrm{ml}) \mathrm{n}=79$ & 17.0 & $2.50-43.7$ \\
\hline
\end{tabular}

Table 2. Median and range values of each blood biomarkers according to their functional group. CRP: C-reactive protein; GPx glutathione peroxidase; GSH: glutathione; ICAM-1: intercellular adhesion molecule-1; mtDNA: mitochondrial DNA; LBP: lipopolysaccharide-binding protein; LPS: lipopolysaccharide; Ox-LDL: oxidized-low-density lipoprotein; PAI-1: plasminogen activator inhibitor-1; SOD: superoxide dismutase; TNF$\alpha$ : tumor necrosis factor- $\alpha$; VCAM-1: vascular cell adhesion molecule-1.

pro-inflammatory cytokines (e.g. TNF- $\alpha$ and IL-6). It is a sensitive marker of systemic inflammation ${ }^{55}$ and has been associated with cardiometabolic risk ${ }^{56}$. Consistent with our results, many studies reported increased plasma CRP in cALL survivors compared to controls ${ }^{7-9,29}$. CRP levels were associated with the MetS in this population ${ }^{8}$.

Our results showed altered adipokine levels in relation to cardiometabolic outcomes. Adjusted model showed that higher leptin-adiponectin ratio was associated with an elevated risk of obesity, insulin resistance and low HDL-C. There are compelling data that emphasize the associations between adiponectin, leptin and cardiometabolic complications in CALL survivors ${ }^{19-21,57-60}$. In adolescent survivors, high leptin-adiponectin ratio was associated with $\mathrm{MetS}^{20}$. Particularly, visceral adiposity was associated with disturbed adipokine balance (i.e., increased leptin and decreased adiponectin $)^{61,62}$, chronic low-grade inflammation ${ }^{63}$ and dyslipidemia as characterized by decreased HDL-C levels. Our results support the reported positive association between plasma adiponectin levels and HDL-C ${ }^{64}$. Adiponectin regulates HDL-C concentration by reducing HDL-C catabolism ${ }^{65}$ and contributes to the inhibition of hepatic lipase activity ${ }^{65}$, an enzyme that hydrolyzes triglycerides and phospholipids contained in HDL particles. In our study, high leptin-adiponectin ratio was associated with increased risk of insulin resistance. In line with our results, HOMA-IR has been associated with higher leptin-adiponectin ratio in both men and women ${ }^{19}$ and in women only ${ }^{58}$.

We also found that high leptin levels were strongly associated with the risk of obesity and insulin resistance. This finding corroborates the presence of long-term hyperleptinemia in cALL survivors ${ }^{19,21}$ that may disrupt communication with the brain and, ultimately, energy usage and insulin signalling ${ }^{66}$. Our results also emphasized the association between low visfatin levels and the risk of insulin resistance. The role of visfatin in insulin resistance is still controversial [reviewed in ${ }^{67}$. Visfatin was reported to have insulin-like activity and to bind the insulin receptor, thereby lowering blood glucose levels ${ }^{68}$. In mice, acute administration of visfatin lowered blood glucose levels ${ }^{68}$. Interestingly, transient overexpression of visfatin enhanced its plasma level, improved insulin sensitivity and had hypocholesterolemic effects in normal-chow rats and rats on high-fat diet ${ }^{69,70}$. However, another study found that visfatin does not have insulin-mimetic effects in mice but rather functions as an extracellular NAD biosynthetic enzyme critical for glucose-stimulated insulin secretion by pancreatic $\beta$-cells ${ }^{71}$. Although studies have provided evidence that visfatin is a biomarker for cALL remission ${ }^{72}$ and bone mineral density of cALL survivors $^{73}$, no prior study had yet examined the relationship between visfatin levels and cardiometabolic risk factors in the context of survivorship. In parallel, we have highlighted the association between high PAI-1 levels, a physiological regulatory protein of the fibrinolytic system, and the risk of obesity in our cohort. In line with our findings, a study reported increased plasma PAI-1 levels in cALL survivors compared to controls ${ }^{74}$, but the association with obesity was not assessed. 


\begin{tabular}{|c|c|c|c|c|c|c|c|c|c|c|}
\hline \multirow[b]{2}{*}{ Biomarker } & \multicolumn{2}{|l|}{ Obesity } & \multicolumn{2}{|l|}{ Insulin resistance } & \multicolumn{2}{|l|}{ Pre-HTN/HTN } & \multicolumn{2}{|l|}{ Dyslipidemia } & \multirow{2}{*}{\begin{tabular}{|l|} 
MetS \\
OR $(95 \% \mathrm{CI})$ \\
\end{tabular}} & \multirow[b]{2}{*}{$p$ value } \\
\hline & OR $(95 \% \mathrm{CI})$ & $p$ value & OR $(95 \% \mathrm{CI})$ & $p$ value & OR $(95 \% \mathrm{CI})$ & $p$ value & OR $(95 \% \mathrm{CI})$ & $p$ value & & \\
\hline & \multicolumn{9}{|l|}{ Crude models } & \\
\hline Adiponectin & $0.27(0.14-0.52)$ & $<0.0001$ & $0.21(0.08-0.53)$ & $<0.001$ & $0.73(0.30-1.81)$ & 0.50 & $0.36(0.20-0.67)$ & 0.001 & $0.08(0.02-0.46)$ & 0.004 \\
\hline Leptin & $5.65(2.76-11.6)$ & $<0.0001$ & $6.09(2.25-16.5)$ & $<0.001$ & $0.41(0.15-1.09)$ & 0.07 & $1.19(0.64-2.23)$ & 0.58 & $1.71(0.61-4.83)$ & 0.32 \\
\hline Ratio leptin:adiponectin & $13.9(6.09-31.7)$ & $<0.0001$ & $16.6(4.29-63.9)$ & $<0.001$ & $0.65(0.26-1.67)$ & 0.37 & $2.20(1.16-4.17)$ & 0.02 & $6.88(1.71-27.7)$ & 0.007 \\
\hline Resistin & $1.04(0.43-2.47)$ & 0.94 & $2.48(0.73-8.43)$ & 0.15 & $1.03(0.31-3.38)$ & 0.97 & $1.81(0.77-4.24)$ & 0.17 & $3.98(0.89-17.8)$ & 0.07 \\
\hline Visfatin & $0.67(0.36-1.28)$ & 0.22 & $0.25(0.10-0.62)$ & 0.003 & $1.26(0.5-3.19)$ & 0.62 & $0.69(0.37-1.29)$ & 0.24 & $0.45(0.16-1.32)$ & 0.15 \\
\hline IL-6 & $1.79(1.04-3.09)$ & 0.04 & $0.90(0.47-1.75)$ & 0.76 & $1.18(0.54-2.58)$ & 0.68 & $1.25(0.74-2.09)$ & 0.40 & $1.80(0.74-4.40)$ & 0.20 \\
\hline TNF- $a$ & $1.86(1.08-3.20)$ & 0.03 & $1.81(0.92-3.56)$ & 0.09 & $1.02(0.48-2.17)$ & 0.96 & $1.10(0.66-1.83)$ & 0.72 & $2.85(1.11-7.37)$ & 0.03 \\
\hline PAI-1 & $3.30(1.64-6.62)$ & $<0.001$ & $3.23(1.29-8.13)$ & 0.01 & $1.66(0.62-4.46)$ & 0.32 & $2.23(1.15-4.33)$ & 0.02 & $2.94(0.93-9.28)$ & 0.07 \\
\hline \multirow[t]{2}{*}{ CRP } & $2.82(1.57-5.07)$ & $<0.001$ & $3.73(1.87-7.47)$ & $<0.001$ & $0.97(0.41-2.28)$ & 0.95 & $2.71(1.52-4.83)$ & $<0.001$ & $5.02(2.03-12.4)$ & $<0.001$ \\
\hline & \multicolumn{9}{|l|}{ Adjusted models } & \\
\hline Adiponectin & $0.18(0.09-0.38)$ & $<0.0001$ & $0.17(0.07-0.45)$ & $<0.001$ & $1.04(0.41-2.66)$ & 0.94 & $0.34(0.18-0.66)$ & 0.002 & $0.07(0.01-0.38)$ & 0.002 \\
\hline Leptin & $9.57(3.41-26.9)$ & $<0.0001$ & $13.2(3.68-47.1)$ & $<0.0001$ & $0.99(0.29-3.36)$ & 0.99 & $1.70(0.72-4.06)$ & 0.23 & $3.21(0.86-11.9)$ & 0.08 \\
\hline Ratio leptin:aiponectin & $15.7(6.20-39.7)$ & $<0.0001$ & $20.6(5.17-82.1)$ & $<0.0001$ & $1.14(0.4-3.14)$ & 0.81 & $2.89(1.34-6.24)$ & 0.007 & $11.2(2.58-48.7)$ & 0.001 \\
\hline Resistin & $0.88(0.34-2.30)$ & 0.80 & $2.26(0.65-7.85)$ & 0.20 & $1.49(0.42-5.32)$ & 0.54 & $1.97(0.74-5.21)$ & 0.17 & $7.40(1.29-42.5)$ & 0.03 \\
\hline Visfatin & $0.74(0.38-1.45)$ & 0.38 & $0.27(0.11-0.66)$ & 0.004 & $0.96(0.36-2.54)$ & 0.94 & $0.66(0.34-1.32)$ & 0.24 & $0.41(0.14-1.25)$ & 0.12 \\
\hline IL-6 & $1.72(0.98-3.02)$ & 0.06 & $0.88(0.45-1.72)$ & 0.71 & $1.23(0.56-2.71)$ & 0.61 & $1.22(0.71-2.10)$ & 0.47 & $1.78(0.73-4.33)$ & 0.21 \\
\hline TNF- $a$ & $2.34(1.31-4.18)$ & 0.004 & $2.13(1.06-4.29)$ & 0.03 & $0.83(0.38-1.81)$ & 0.65 & $1.22(0.71-2.10)$ & 0.46 & $3.40(1.29-8.94)$ & 0.01 \\
\hline PAI-1 & $3.37(1.61-7.05)$ & 0.001 & $3.14(1.25-7.90)$ & 0.02 & $1.78(0.64-4.96)$ & 0.27 & $1.99(0.98-4.04)$ & 0.06 & $2.40(0.76-7.52)$ & 0.13 \\
\hline CRP & $2.21(1.19-4.09)$ & 0.01 & $3.27(1.58-6.79)$ & 0.002 & $1.53(0.61-3.82)$ & 0.37 & $2.61(1.40-4.86)$ & 0.003 & $6.52(2.38-17.9)$ & $<0.001$ \\
\hline
\end{tabular}

Table 3. Associations between biomarkers of inflammation and cardiometabolic complications in survivors of childhood acute lymphoblastic leukemia: crude and adjusted models. The crude and adjusted models were assessed between each biomarker and each cardiometabolic outcome. Models were adjusted for CRT exposure, age at diagnosis, time since diagnosis and sex. Odds ratio (non-corrected 95\% CI) and p-value are indicated for each association. Significant associations are in boldface. Bonferroni-adjusted alpha $=0.05 /$ number of biomarkers $=0.05 / 9=0.006$. Metabolic syndrome was defined according to the International Diabetes Federation. CI: confidence interval; CRP: C-reactive protein; CRT: cranial radiotherapy; HTN: arterial hypertension; IL-6: interleukin-6; MetS: metabolic syndrome; PAI-1: plasminogen activator inhibitor-1; TNF$\alpha$ : tumor necrosis factor- $\alpha$.

\begin{tabular}{|c|c|c|c|c|c|c|c|c|c|c|}
\hline \multirow[b]{2}{*}{ Biomarker } & \multicolumn{2}{|l|}{ Obesity } & \multicolumn{2}{|l|}{ Insulin resistance } & \multicolumn{2}{|l|}{ Pre-HTN/HTN } & \multicolumn{2}{|l|}{ Dyslipidemia } & \multicolumn{2}{|l|}{ MetS } \\
\hline & OR $(95 \% \mathrm{CI})$ & $p$ value & OR $(95 \% \mathrm{CI})$ & $p$ value & OR (95\% CI) & $p$ value & OR $(95 \% \mathrm{CI})$ & $p$ value & OR $(95 \% \mathrm{CI})$ & $p$ value \\
\hline & \multicolumn{10}{|l|}{\begin{tabular}{|l|} 
Crude models \\
\end{tabular}} \\
\hline GSH & $0.55(0.32-0.95)$ & 0.03 & $0.71(0.37-1.39)$ & 0.32 & $1.08(0.50-2.33)$ & 0.85 & $0.79(0.47-1.32)$ & 0.36 & $1.35(0.56-3.29)$ & 0.51 \\
\hline GPx & $0.83(0.49-1.42)$ & 0.50 & $0.80(0.41-1.55)$ & 0.50 & $1.57(0.73-3.38)$ & 0.25 & $1.78(1.06-2.97)$ & 0.03 & $1.22(0.52-2.91)$ & 0.65 \\
\hline Ox-LDL & $1.31(0.70-2.44)$ & 0.40 & $1.39(0.63-3.06)$ & 0.41 & $0.82(0.32-2.05)$ & 0.66 & $7.89(3.97-15.7)$ & $<0.0001$ & $3.08(1.00-9.55)$ & 0.05 \\
\hline Protein carbonyls & $1.04(0.43-2.47)$ & 0.94 & $1.72(0.53-5.58)$ & 0.37 & $0.49(0.14-1.69)$ & 0.26 & $1.26(0.54-2.92)$ & 0.60 & $1.52(0.42-5.57)$ & 0.53 \\
\hline SOD & $1.24(0.65-2.36)$ & 0.52 & $1.09(0.49-2.42)$ & 0.84 & $0.80(0.32-2.03)$ & 0.64 & $0.90(0.48-1.69)$ & 0.75 & $0.76(0.27-2.10)$ & 0.59 \\
\hline \multirow[t]{2}{*}{ mtDNA } & $1.14(0.65-2.00)$ & 0.64 & $0.89(0.44-1.80)$ & 0.74 & $0.86(0.39-1.89)$ & 0.71 & $1.09(0.64-1.86)$ & 0.75 & $1.59(0.61-4.17)$ & 0.34 \\
\hline & \multicolumn{10}{|l|}{ Adjusted models } \\
\hline GSH & $0.51(0.29-0.91)$ & 0.02 & $0.72(0.36-1.42)$ & 0.34 & $1.35(0.61-2.99)$ & 0.46 & $0.88(0.51-1.51)$ & 0.65 & $1.62(0.65-4.00)$ & 0.30 \\
\hline GPx & $0.84(0.48-1.46)$ & 0.53 & $0.80(0.41-1.57)$ & 0.52 & $1.56(0.71-3.43)$ & 0.27 & $1.98(1.15-3.42)$ & 0.01 & $1.30(0.55-3.09)$ & 0.55 \\
\hline Ox-LDL & $1.36(0.71-2.62)$ & 0.35 & $1.36(0.61-3.02)$ & 0.46 & $0.66(0.25-1.71)$ & 0.39 & $7.90(3.80-16.4)$ & $<0.0001$ & $2.87(0.93-8.86)$ & 0.07 \\
\hline Protein carbonyls & $0.72(0.28-1.90)$ & 0.51 & $1.42(0.42-4.78)$ & 0.58 & $0.57(0.16-2.10)$ & 0.40 & $1.02(0.40-2.63)$ & 0.96 & $1.73(0.42-7.20)$ & 0.45 \\
\hline SOD & $1.11(0.55-2.21)$ & 0.78 & $0.97(0.42-2.23)$ & 0.94 & $1.17(0.43-3.17)$ & 0.76 & $0.88(0.43-1.78)$ & 0.72 & $0.82(0.29-2.39)$ & 0.72 \\
\hline mtDNA & $1.27(0.71-2.25)$ & 0.43 & $0.97(0.48-1.98)$ & 0.94 & $0.78(0.35-1.75)$ & 0.55 & $1.22(0.70-2.13)$ & 0.49 & $1.82(0.69-4.77)$ & 0.22 \\
\hline
\end{tabular}

Table 4. Associations between biomarkers of oxidative stress and cardiometabolic complications in survivors of childhood acute lymphoblastic leukemia: crude and adjusted models. The crude and adjusted models were assessed between each biomarker and each cardiometabolic outcome. Models were adjusted for CRT exposure, age at diagnosis, time since diagnosis and sex. Odds ratio (non-corrected 95\% CI) and p-value are indicated for each association. Significant associations are in boldface. Bonferroni-adjusted alpha $=0.05 /$ number of biomarkers $=0.05 / 6=0.008$. Metabolic syndrome was defined according to the International Diabetes Federation. CI: confidence interval; CRT: cranial radiotherapy; GPx: glutathione peroxidase; GSH: glutathione; HTN: arterial hypertension; MetS: metabolic syndrome; mtDNA: mitochondrial DNA; Ox-LDL: oxidized lowdensity lipoprotein; SOD: superoxide dismutase. 


\begin{tabular}{|c|c|c|c|c|c|c|c|c|c|c|}
\hline \multirow[b]{2}{*}{ Biomarker } & \multicolumn{2}{|l|}{ Obesity } & \multicolumn{2}{|l|}{ Insulin resistance } & \multicolumn{2}{|l|}{ Pre-HTN/HTN } & \multicolumn{2}{|l|}{ Dyslipidemia } & \multicolumn{2}{|l|}{ MetS } \\
\hline & OR (95\% CI) & $p$ value & OR $(95 \%$ CI $)$ & $p$ value & OR $(95 \% \mathrm{CI})$ & $p$ value & OR $(95 \% \mathrm{CI})$ & $p$ value & OR $(95 \% \mathrm{CI})$ & $p$ value \\
\hline & \multicolumn{10}{|l|}{\begin{tabular}{|l|} 
Crude models \\
\end{tabular}} \\
\hline LPS & $0.60(0.29-1.24)$ & 0.17 & $0.91(0.36-2.31)$ & 0.85 & $0.88(0.31-2.54)$ & 0.82 & $0.80(0.40-1.62)$ & 0.53 & $0.39(0.11-1.45)$ & 0.16 \\
\hline \multirow[t]{2}{*}{ LBP } & $2.59(1.48-4.51)$ & 0.001 & $2.26(1.13-4.51)$ & 0.021 & $0.74(0.35-1.59)$ & 0.45 & $2.18(1.30-3.67)$ & 0.003 & $2.75(1.07-7.11)$ & 0.04 \\
\hline & \multicolumn{4}{|l|}{ Adjusted models } & & & & & & \\
\hline LPS & $0.58(0.27-1.25)$ & 0.16 & $0.88(0.34-2.27)$ & 0.80 & $0.90(0.29-2.75)$ & 0.85 & $0.83(0.39-1.77)$ & 0.62 & $0.44(0.12-1.59)$ & 0.21 \\
\hline LBP & $2.03(1.13-3.65)$ & 0.019 & $1.77(0.86-3.66)$ & 0.12 & $0.99(0.44-2.23)$ & 1.00 & $1.92(1.09-3.37)$ & 0.024 & $2.60(0.96-7.06)$ & 0.06 \\
\hline
\end{tabular}

Table 5. Associations between biomarkers of endotoxemia and cardiometabolic complications in survivors of childhood acute lymphoblastic leukemia: crude and adjusted models. The crude and adjusted models were assessed between each biomarker and each cardiometabolic outcome. Models were adjusted for CRT exposure, age at diagnosis, time since diagnosis and sex. Odds ratio (non-corrected 95\% CI) and p-value are indicated for each association. Significant associations are in boldface. Bonferroni-adjusted alpha $=0.05 /$ number of biomarkers $=0.05 / 2=0.025$. Metabolic syndrome was defined according to the International Diabetes Federation. CI: confidence interval; CRT: cranial radiotherapy; HTN: arterial hypertension; LBP: lipopolysaccharide-binding protein; LPS: lipopolysaccharide; MetS: metabolic syndrome.

\begin{tabular}{|c|c|c|c|c|c|c|c|c|c|c|}
\hline \multirow[b]{2}{*}{ Biomarker } & \multicolumn{2}{|l|}{ Obesity } & \multicolumn{2}{|l|}{ Insulin resistance } & \multicolumn{2}{|l|}{ Pre-HTN/HTN } & \multicolumn{2}{|l|}{ Dyslipidemia } & \multicolumn{2}{|l|}{ MetS } \\
\hline & OR (95\% CI) & $p$ value & OR $(95 \% \mathrm{CI})$ & $p$ value & OR $(95 \% \mathrm{CI})$ & $p$ value & OR $(95 \% \mathrm{CI})$ & $p$ value & OR $(95 \% \mathrm{CI})$ & $p$ value \\
\hline & \multicolumn{10}{|l|}{ Crude models } \\
\hline ICAM-1 & $2.11(1.22-3.65)$ & 0.007 & $2.00(1.01-3.96)$ & 0.05 & $1.00(0.47-2.13)$ & 1.000 & $1.83(1.10-3.07)$ & 0.02 & $3.51(1.30-9.53)$ & 0.014 \\
\hline VCAM-1 & $0.60(0.35-1.02)$ & 0.06 & $0.63(0.32-1.24)$ & 0.18 & $1.16(0.54-2.47)$ & 0.70 & $0.58(0.35-0.98)$ & 0.04 & $0.83(0.35-1.98)$ & 0.68 \\
\hline \multirow[t]{2}{*}{ E-Selectin } & $2.44(0.96-6.20)$ & 0.06 & $1.03(0.31-3.42)$ & 0.96 & $0.71(0.21-2.40)$ & 0.58 & $1.42(0.59-3.47)$ & 0.44 & $1.62(0.44-6.00)$ & 0.47 \\
\hline & \multicolumn{10}{|l|}{ Adjusted models } \\
\hline ICAM-1 & $2.15(1.21-3.82)$ & 0.009 & $1.91(0.95-3.84)$ & 0.07 & $1.09(0.50-2.38)$ & 0.81 & $1.82(1.06-3.13)$ & 0.03 & $3.60(1.32-9.84)$ & 0.013 \\
\hline VCAM-1 & $0.86(0.47-1.57)$ & 0.62 & $0.84(0.40-1.77)$ & 0.65 & $0.81(0.35-1.88)$ & 0.62 & $0.79(0.44-1.42)$ & 0.43 & $1.30(0.50-3.40)$ & 0.583 \\
\hline E-Selectin & $3.41(1.15-10.09)$ & 0.03 & $1.10(0.33-3.72)$ & 0.88 & $0.55(0.15-2.04)$ & 0.37 & $1.65(0.60-4.57)$ & 0.33 & $1.59(0.40-6.25)$ & 0.511 \\
\hline
\end{tabular}

Table 6. Associations between biomarkers of endothelial function and cardiometabolic complications in survivors of childhood acute lymphoblastic leukemia: crude and adjusted models. The crude and adjusted models were assessed between each biomarker and each cardiometabolic outcome. Models were adjusted for CRT exposure, age at diagnosis, time since diagnosis and sex. Odds ratio (non-corrected 95\% CI) and p-value are indicated for each association. Bonferroni-adjusted alpha $=0.05 /$ number of biomarkers $=0.05 / 3=0.017$. Metabolic syndrome was defined according to the International Diabetes Federation. CI: confidence interval; CRT: cranial radiotherapy; HTN: arterial hypertension; ICAM-1: intercellular adhesion molecule-1; MetS: metabolic syndrome; VCAM-1: vascular cell adhesion molecule-1.

Clinical studies have demonstrated that serum LPS levels were closely related to obesity ${ }^{75-77}$ and insulin resistance ${ }^{76,78,79}$. While our study did not reveal an association, we cannot exclude that this negative result could be the result of the short half-life of LPS ${ }^{80}$. LBP is considered a biomarker of plasma endotoxin exposure because its half-life $(24 \mathrm{~h})$ is longer than of LPS $(<8 \mathrm{~min} \text { in mice and up to } 3 \mathrm{~h} \text { in humans })^{81}$. Hence, it has been used as a proxy to assess chronic endotoxemia ${ }^{82}$. We found that higher LBP levels were associated with an increased risk of obesity, insulin resistance and low HDL-C in cALL survivors. LBP participates in the LPS-mediated inflammatory response, facilitating the binding of LPS to toll-like receptor 4 (TLR4) ${ }^{83}$. TLR4 activation leads to increase transcription pro-inflammatory genes that promote the initiation of cytokine signalling cascades ${ }^{83}$, reactive oxygen species production and insulin desensitization ${ }^{84}$. TLR4 activation by LPS and LBP complex in insulin target cells can directly dampen insulin action through mechanisms involving cytokine and pro-inflammatory kinases JNK, IKK and $\mathrm{p} 38^{84}$. LBP also acts as a lipid transfer protein by catalysing the transfer of LPS-sCD14 complexes to HDL particles ${ }^{85}$. Our findings corroborate other studies in which LBP levels were strongly associated with lipid abnormalities ${ }^{75,86-89}$. They are also in agreement with two prospective studies showing associations between higher LBP levels and the risk of insulin resistance and impaired fasting glucose in men ${ }^{90}$, and with the risk of MetS in men and women ${ }^{89}$. Similarly, cross-sectional studies highlighted a relationship between higher LBP levels and insulin resistance in adolescent men and women ${ }^{91}$ or in adult men ${ }^{75,92}$. Also, in our study, the SEM analysis supports that the relationships between endotoxemia and obesity, insulin resistance, dyslipidemia and MetS are mediated by inflammation. This indicates that circulating LBP is a relevant biomarker for systemic inflammation in CALL survivors.

Among the biomarkers of OxS examined, associations were found only for ox-LDL with dyslipidemia and having low HDL-C. There has been a growing body of evidence suggesting that plasma ox-LDLs are increased in pathologic conditions linked to cardiovascular diseases including insulin resistance, obesity and MetS $S^{93,94}$. As for endothelial function, only ICAM-1 levels were associated with the risk of obesity and MetS. A recent study reported increased plasma ICAM-1 levels in 64 cALL survivors (median age of 15 years) compared to 


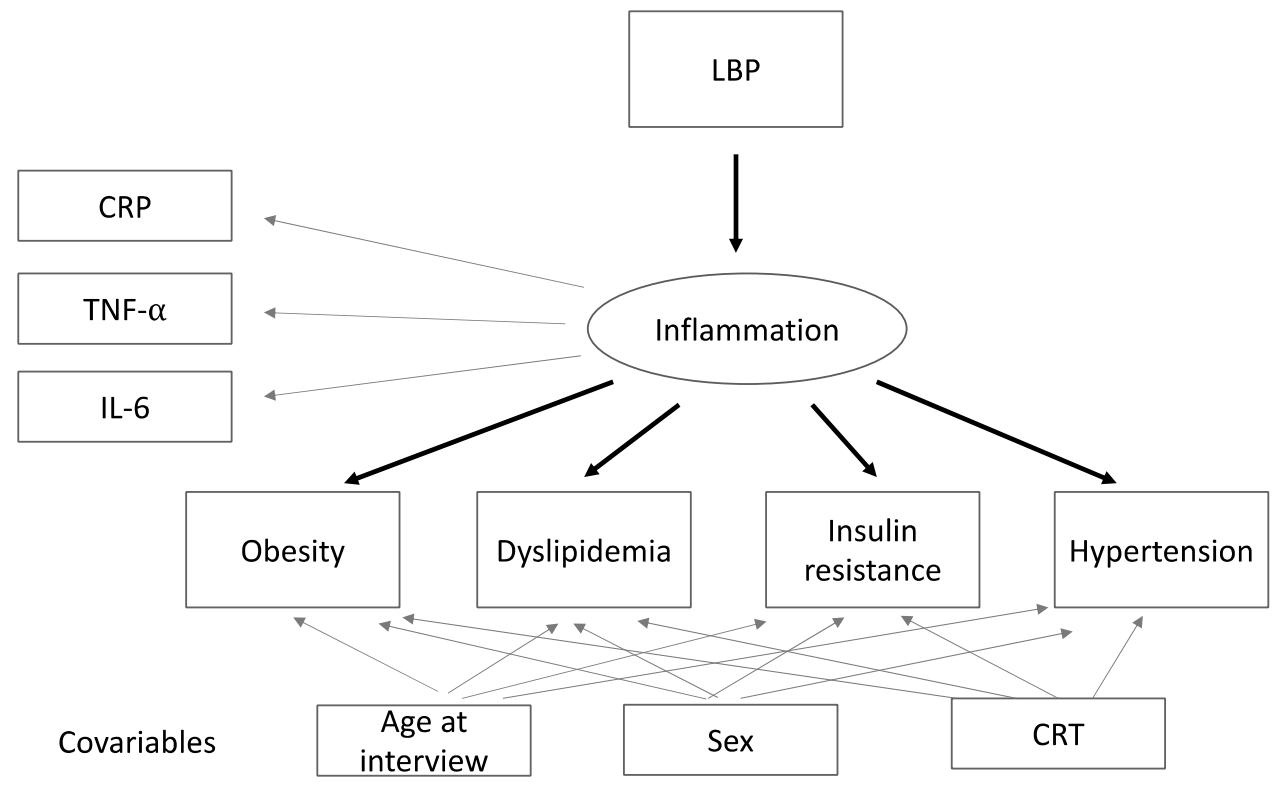

Figure 1. Path diagram corresponding to structural equation model between LBP (biomarker of endotoxemia), inflammation as the latent variable (derived from CRP, TNF- $\alpha$ and IL-6) and cardiometabolic outcomes. The conventional rules of SEM visualization were applied.

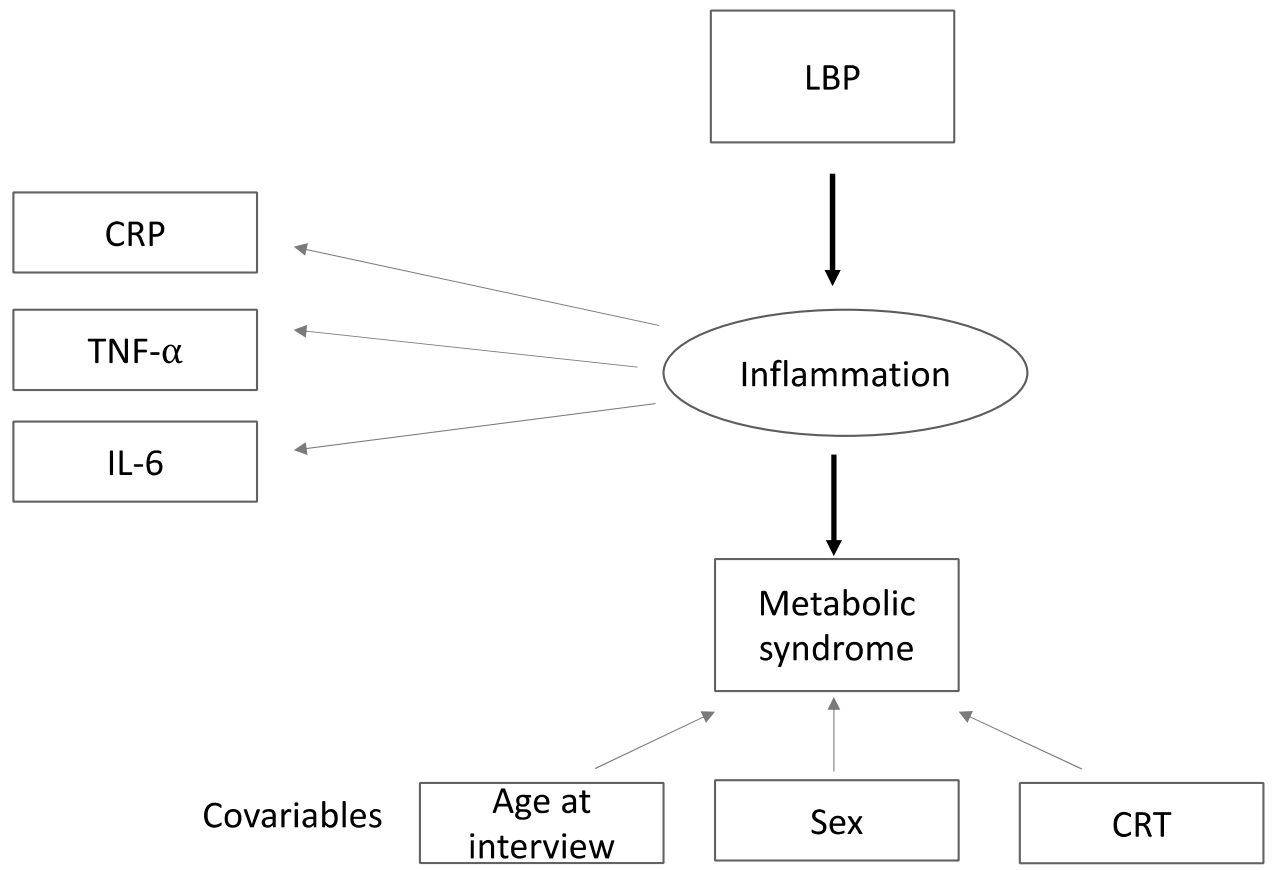

Figure 2. Path diagram corresponding to structural equation model between LBP (biomarker of endotoxemia), inflammation as the latent variable (derived from CRP, TNF- $\alpha$ and IL-6) and metabolic syndrome. The conventional rules of SEM visualization were applied.

36 controls $^{29}$. Conversely to our findings, a small study by Barbosa-Cortés et al. did not find any association between circulating ICAM-1 levels and the prevalence of the MetS in cALL or lymphoma survivors ${ }^{20}$. These discrepancies with our study may be due, in part, to different patients' characteristics (median age of 12.1 vs. 21.8 years), CRT exposure ( 32.7 vs. $59.4 \%$ ) and number of participants with MetS $(n=7$ vs. $n=22)$. As a matter of fact, it was reported that ICAM-1 levels can be influenced by age, insulin resistance and other inflammatory conditions ${ }^{95}$. We did not report associations between VCAM-1 or E-selectin and cardiometabolic complications. Elsewhere, a small study comprising of 27 cALL survivors (median age of 20 years) reported high VCAM-1 levels compared to 20 controls $^{30}$, but the association with cardiometabolic complications was not investigated. In the general population, no evidence of a positive association of VCAM-1 and cardiometabolic outcomes 


\begin{tabular}{|c|c|c|c|}
\hline & \multirow{2}{*}{$\begin{array}{l}\text { Non standardized estimates } \\
\text { Estimate }(95 \% \mathrm{CI})\end{array}$} & \multicolumn{2}{|l|}{ Standardized estimates } \\
\hline & & Estimate $(95 \% \mathrm{CI})$ & $p$ value \\
\hline \multicolumn{4}{|c|}{ Loadings of the biomarkers CRP, TNF- $\alpha$ and IL- 6 for the latent variable inflammation } \\
\hline CRP & 1.000 & $0.871(0.761,0.981)$ & $<0.001$ \\
\hline TNF- $a$ & $0.012(-0.087,0.110)$ & $0.012(-0.086,0.109)$ & 0.82 \\
\hline IL-6 & $0.060(0.031,0.089)$ & $0.345(0.219,0.472)$ & $<0.001$ \\
\hline \multicolumn{4}{|c|}{ Regression of inflammation on LBP } \\
\hline LBP & $0.304(0.245,0.363)$ & $0.714(0.604,0.823)$ & $<0.001$ \\
\hline \multicolumn{4}{|c|}{ Regression of obesity on inflammation, sex, age at interview, CRT } \\
\hline Inflammation & $0.097(0.041,0.153)$ & $0.324(0.216, .552)$ & $<0.001$ \\
\hline Sex (M vs. F) & $-0.248(-0.607,0.111)$ & $-0.117(-0.278,0.049)$ & 0.17 \\
\hline Age at interview & $0.009(-0.019,0.038)$ & $0.056(-0.113,0.223)$ & 0.52 \\
\hline CRT (yes vs. no) & $0.185(-0.198,0.567)$ & $0.086(-0.088,0.256)$ & 0.34 \\
\hline \multicolumn{4}{|c|}{ Regression of dyslipidemia on inflammation, sex, age at interview, CRT } \\
\hline Inflammation & $0.082(0.024,0.139)$ & $0.274(0.099,0.448)$ & $<0.01$ \\
\hline Sex (M vs. F) & $0.197(-0.168,0.561)$ & $0.093(-0.079,0.266)$ & 0.29 \\
\hline Age at interview & $0.031(0.001,0.062)$ & $0.188(0.011,0.365)$ & 0.04 \\
\hline CRT (yes vs. no) & $0.125(-0.247,0.498)$ & $0.058(-0.115,0.231)$ & 0.51 \\
\hline \multicolumn{4}{|c|}{ Regression of insulin resistance on inflammation, sex, age at interview, CRT } \\
\hline Inflammation & $0.115(0.056,0.173)$ & $0.381(0.212,0.549)$ & $<0.001$ \\
\hline Sex (M vs. F) & $-0.127(-0.544,0.290)$ & $-0.060(-0.255,0.135)$ & 0.55 \\
\hline Age at interview & $0.006(-0.032,0.044)$ & $0.037(-0.187,0.261)$ & 0.75 \\
\hline CRT (yes vs. no) & $0.253(-0.180,0.686)$ & $0.117(-0.079,0.313)$ & 0.24 \\
\hline \multicolumn{4}{|c|}{ Regression of pre-HTN/HTN on inflammation adjusted for sex, age at interview, CRT } \\
\hline Inflammation & $0.014(-0.085,0.113)$ & $0.046(-0.276,0.367)$ & 0.78 \\
\hline Sex (M vs. F) & $0.801(0.239,1.363)$ & $0.371(0.142,0.600)$ & $<0.01$ \\
\hline Age at interview & $-0.007(-0.050,0.035)$ & $-0.043(-0.291,0.205)$ & 0.73 \\
\hline CRT (yes vs. no) & $0.234(-0.270,0.737)$ & $0.106(-0.121,0.333)$ & 0.36 \\
\hline
\end{tabular}

Table 7. SEM estimates of the associations between LBP, inflammation, obesity, dyslipidemia, insulin resistance and pre-HTN/HTN. CRT: cranial radiotherapy; CFI: Comparative Fit Index; HTN: hypertension; LBP: LPS binding protein; RMSEA: Root Mean Square Error of Approximation; SRMR: Standardized Root Mean Square Residual; TLI: Tucker-Lewis Index. $p$ values of non-standardized estimates are not shown as they are almost identical to $p$ values of standardized estimates.

\begin{tabular}{|c|c|c|c|}
\hline & \multirow{2}{*}{$\begin{array}{l}\text { Non standardized estimates } \\
\text { Estimate }(95 \% \mathrm{CI})\end{array}$} & \multicolumn{2}{|l|}{ Standardized estimates } \\
\hline & & Estimate $(95 \% \mathrm{CI})$ & $p$ value \\
\hline \multicolumn{4}{|c|}{$\begin{array}{l}\text { Loadings of the biomarkers CRP, TNF- } \alpha \text { and IL- } 6 \text { for the latent variable inflamma- } \\
\text { tion }\end{array}$} \\
\hline CRP & 1.000 & $0.944(0.767,1.122)$ & $<0.001$ \\
\hline TNF- $\alpha$ & $0.002(-0.072,0.076)$ & $0.002(-0.076,0.081)$ & 0.95 \\
\hline IL-6 & $0.051(0.019,0.083)$ & $0.322(0.181,0.463)$ & $<0.001$ \\
\hline \multicolumn{4}{|c|}{ Regression of inflammation on LBP } \\
\hline LBP & $0.297(0.238,0.355)$ & $0.649(0.502,0.796)$ & $<0.001$ \\
\hline \multicolumn{4}{|c|}{$\begin{array}{l}\text { Regression of metabolic syndrome on inflammation adjusted for sex, age at inter- } \\
\text { view, CRT }\end{array}$} \\
\hline Inflammation & $0.099(0.031,0.168)$ & $0.345(0.153,0.537)$ & $<0.001$ \\
\hline Sex (M vs. F) & $0.397(-0.143,0.937)$ & $0.182(-0.055,0.418)$ & 0.13 \\
\hline Age at interview & $0.045(-0.004,0.093)$ & $0.254(-0.009,0.517)$ & 0.06 \\
\hline CRT (yes vs. no) & $0.131(-0.513,0.774)$ & $0.059(-0.230,0.347)$ & 0.69 \\
\hline \multicolumn{4}{|c|}{$\begin{array}{l}\text { Goodness of fit measures: } \mathrm{CFI}=1.000, \mathrm{TLI}=1.042, \mathrm{RMSEA}(90 \% \mathrm{CI})=0.000(0.000 \text {, } \\
0.043) \text {, SRMR }=0.025\end{array}$} \\
\hline
\end{tabular}

Table 8. SEM estimates of the associations between LBP, inflammation and metabolic syndrome. CRT: cranial radiotherapy; CFI: Comparative Fit Index; LB: LPS binding protein; RMSEA: Root Mean Square Error of Approximation; SRMR: Standardized Root Mean Square Residual; TLI: Tucker-Lewis Index. $p$ values of nonstandardized estimates are not shown as they almost identical to $p$ values of standardized estimates. 
has been reported ${ }^{96-98}$. However, it was suggested that VCAM-1 is expressed primarily at an advanced stage of atherosclerosis ${ }^{98}$ whereas ICAM-1 is a general marker of a pro-inflammatory state in healthy population. Besides, E-selectin was found correlated with obesity and insulin resistance among obese subjects ${ }^{99,100}$ and to be a good predictor for insulin resistance in women ${ }^{101}$. In the context of cALL survivorship, no prior study has examined the relationship between E-selectin and cardiometabolic complications, despite the important roles played by this protein in endothelial function.

Finally, no significant association was found between the biomarkers and hypertension. Conversely, two studies in cALL and lymphoma survivors found that low levels of adiponectin were associated with hypertension ${ }^{20,22}$. These different findings could be explained by the fact that our study was restricted to survivors of cALL and by the low prevalence of hypertension in our cohort (12\%) compared to the others (27\% and 54\%).

The strengths of our study include a broad panel of blood biomarkers of endotoxemia, inflammation, oxidative stress and endothelial function in a well-characterized cohort of cALL survivors. We used the SEM, a multivariate statistical analysis technique, in addition to the conventional regression analysis. This allows to simultaneously test the relationships between the different variables potentially explaining the development of the cardiometabolic complications. Limitations include the monocentric study design and the absence of a healthy control group. Additionally, as our study population was Caucasian, our results may not be generalizable to other ethnic groups as, in epidemiological studies, plasma levels of inflammatory biomarkers associated with cardiometabolic diseases can differ by ethnic group ${ }^{102}$. Although we did adjust a priori for four selected confounding variables, residual confounding by other factors may bias the results. The modest size of our cohort ( $n=246$, corresponding to the maximal sample size) may have limited our ability to detect weak associations, especially given that sample sizes available for analyses depended on biomarkers. In general, we acknowledge that our study may have generated both false-negative and false-positive findings and will need to be replicated in larger samples. Since structural equation modeling is very sensible to sample $\operatorname{size}^{103}$ our results should be interpreted with caution. Furthermore, there is no cutoff for fit indices to evaluate the goodness of fit for a model including binary variables ${ }^{104,105}$. Hence, the cutoff used to evaluate the goodness of fit of our models were defined for models with continuous variables, a limit that has to be considered.

\section{Conclusions}

This study revealed significant associations between plasma biomarkers of visceral inflammation, endotoxemia and endothelial function and late occurring cardiometabolic adverse effects in cALL survivors. It also highlights the relationship between LBP, a protein related to metabolic endotoxemia, inflammation and the presence of cardiometabolic complications. Identification of biomarkers and biological mechanisms could open new avenues for prevention strategies to minimize the long-term sequelae, improve patient follow-up and ultimately optimize the quality of life of this high-risk population.

\section{Material and methods}

Study population and protocol. Participants survivors of cALL included in this study $(\mathrm{n}=246)$ were recruited as part of the PETALE study (Sainte-Justine University Health Center (SJUHC), Quebec, Canada). The study design and cohort characteristics are described $\mathrm{in}^{106}$. Briefly, subjects enrolled in the PETALE study were treated for CALL at SJUHC with the Dana Farber Cancer Institute protocols 87-01 to 05-01 107 . Survivors less than 19 years old at diagnosis, more than 5 years post diagnosis and free of relapse were invited to participate. At interview, participants completed a core laboratory assessment as well as anthropometric and clinical evaluations. Demographic characteristics relevant for the following analyses are outlined in Table 1 . The study was approved by the Institutional Review Board of SJUHC and investigations were carried out in accordance with the principles of the Declaration of Helsinki. Written informed consent was obtained from study participants or parents/guardians.

Assessment of cardiometabolic complications. Participants were assessed for cardiometabolic outcomes, specifically obesity, insulin resistance, pre-HTN and HTN and dyslipidemia (cut-off values presented in Supplementary Table S1). The prevalence of cardiometabolic complications in this cohort has been previously described ${ }^{4}$. Briefly, obesity was determined by the presence of at least one of two factors: obese according to body mass index $(\mathrm{BMI})^{108,109}$ and/or high waist circumference ${ }^{110,111}$. Insulin resistance measured in fasting plasma and defined by the presence of at least two of three factors: glucose $>6.1 \mathrm{mmol} / \mathrm{L}$; and/or; glycated hemoglobin $>6.0 \%$; and/or; homeostasis model assessment [HOMA-IR, insulin $(\mathrm{mIU} / \mathrm{L}) \times$ glucose $(\mathrm{mmol} / \mathrm{L}) / 22.5)] \geq 2.86(\text { adults })^{112}$ or $\geq 95$ th percentile (children $)^{113}$. Pre-HTN and HTN were determined according to current recommendations in adults (normal <130/85 mmHg; pre-HTN: $\geq 130 / 85$ and $<140 / 90 \mathrm{mmHg}$; HNN $\geq 140 / 90 \mathrm{mmHg}$ ) ${ }^{114}$ and in children (normal: $<90$ th percentile; pre-HTN: $\geq 90$ th and $<95$ th percentile and HTN $\geq 95$ th percentile according to age and height $)^{115}$. Participants who were taking drugs to treat hypertension were also considered hypertensive. Dyslipidemia was determined in fasting plasma by the presence of at least one of three factors: high LDL-C; high triglycerides and/or low HDL-C according to cut-off values for age and sex ${ }^{116,117}$. MetS was defined according to the International Diabetes Federation ${ }^{118}$. For participants 16 years and older, we considered: having waist circumference $\geq 94 \mathrm{~cm}$ in men and $\geq 80 \mathrm{~cm}$ in women, plus any two of the following factors: (i) triglycerides $\geq 1.70 \mathrm{mmol} / \mathrm{L}$ or on drug treatment; (ii) HDL-C $<1.03 \mathrm{mmol} / \mathrm{L}$ in men and $<1.3 \mathrm{mmol} / \mathrm{L}$ in women or on therapy; (iii) systolic $\geq 130 \mathrm{mmHg}$ or diastolic $\geq 85 \mathrm{mmHg}$ or on treatment and; (iv) fasting glucose $\geq 5.6 \mathrm{mmol} / \mathrm{L}$. For children 10 to 16 years, MetS was defined by waist circumference $\geq 90$ th percentile plus any two of: (i) triglycerides $\geq 1.70 \mathrm{mmol} / \mathrm{L}$; (ii) $\mathrm{HDL}-\mathrm{C}<1.03 \mathrm{mmol} / \mathrm{L}$; (iii) systolic $\geq 130 \mathrm{mmHg}$ or diastolic $\geq 85 \mathrm{mmHg}$ and; (iv) fasting glucose $\geq 5.6 \mathrm{mmol} / \mathrm{L}$. 
Quantification of biomarkers. Overnight fasting peripheral blood samples were collected in EDTA tubes that were kept on ice until centrifugation. Plasma was separated by low speed centrifugation $(2200 g, 20 \mathrm{~min})$ at $4{ }^{\circ} \mathrm{C}$ within $45 \mathrm{~min}$ of collection and stored at $-80^{\circ} \mathrm{C}$ until analysis. White blood cells were isolated and stored at $-80^{\circ} \mathrm{C}$ until analysis. Red blood cells (RBCs) were washed with saline, butylated hydroxytoluene $(10 \mu \mathrm{l} / \mathrm{ml})$ (Sigma-Aldrich, St. Louise, MO, USA) was added and RBCs were stored at $-80^{\circ} \mathrm{C}$ until analysis.

Biomarkers of inflammation. Commercial ELISA kits were used to measure the following biomarkers in fasting plasma: adiponectin (\#BMS2032), resistin (\#BMS2040) and plasminogen activator inhibitor-1 (PAI-1) (\#BMS2033) (Thermo Fisher Scientific, Waltham, MA, USA), leptin (\# EZHL-80SK, EMD Millipore, Burlington, MA, USA) and visfatin (\#EIA-VIS, RayBiotech, GA, USA). High sensitivity CRP was measured in fasting serum by immunoturbidimetry. IL- 6 and TNF- $\alpha$ were measured in fasting plasma using the multiplex ELISA V-Plex Pro-inflammatory Panel I according to the manufacturer's instructions (MesoScale Discovery, Rockville, MD, USA).

Biomarkers of oxidative stress. SOD activity was measured in plasma by the method of xanthine/xanthine oxidase using kits from Caymen Chemical (\#706002, Ann Arbor, MI, USA). GPx activity was measured in RBCs by measuring consumed NADPH using kits from Caymen Chemical (\#703102, Ann Arbor, MI, USA). Ox-LDL and protein carbonyls were measured using ELISA kits from Mercodia AB (\#10-1143-01, Uppsala, Sweden) and Cell Biolabs Inc. (\#STA-310, San Diego, CA, USA), respectively. Total glutathione was measured in RBCs by spectrophotometry with the GSH recycling method ${ }^{119,120}$. The intra-assay CVs for SOD, GPx, Ox-LDLs, GSH were $24.2 \%, 11.4 \%, 9.5 \%$ and $10.8 \%$, respectively. Mitochondrial DNA was determined by Quantitative realtime PCR. Genomic DNA was extracted from white blood cells using the Purelink Genomic DNA kit (Thermo Fisher Scientific) following manufacturer's instructions. DNA was quantified using a spectrophotometer and concentrations were adjusted to $10 \mathrm{ng} / \mathrm{ml}$. qPCR for human mitochondria-encoded NADH dehydrogenase 1 (MT-ND1) and hemoglobin subunit beta $(H B B)$ expression analysis was performed using Taqman gene expression probes \#Hs02596873_s1 and \#Hs00758889_s1, respectively. Transcript expression was determined with the 7500 Fast Real-Time PCR System (Applied Biosystems, Foster City, CA, USA). Expression levels were measured by Relative Quantity (RQ, $2^{-\Delta \Delta \mathrm{Ct}}$ ), where $H B B$ expression served as endogenous control for normalization ${ }^{121}$.

Biomarkers of endotoxemia. Levels of LPS and LBP were measured in fasting plasma using commercial ELISA kits from Elabscience (\#E-EL-0025, Houston, TX, USA) and Cell Sciences (\#CKM043, Newburyport, MA, USA), respectively. The intra-assay CVs for LPS and LBP were $15.7 \%$ and $8.1 \%$, respectively.

Biomarkers of endothelial function. Levels of E-selectin were measured using kits from RayBiotech (\#ELHEselectin, Norcross, GA, USA). ICAM-1 and VCAM-1 were measured in fasting plasma with V-PLEX assay kits (Meso-Scale Discovery, Rockville, MD, USA). All experiments were achieved following manufacturers' protocols and all readings were performed using the DTX800/800 Multimode microplate reader (Beckman Coulter, Brea, CA, USA).

Statistical analyses. The biomarkers were dichotomized by the median, with the exception of CRP that was dichotomized by comparing normal $(\leq 3 \mathrm{mg} / \mathrm{L})$ to high $(>3 \mathrm{mg} / \mathrm{L})$ levels. The association was assessed between each biomarker and each cardiometabolic outcome using a logistic regression analysis with the targeted biomarker as the independent variable. Analyses were performed without adjustment (crude models) and with adjustment for CRT exposure, age at diagnosis, time since diagnosis and sex (adjusted models). Firth's penalized maximum likelihood estimation procedure was used to mitigate problems of quasi-complete separation of data points encountered in standard logistic regression analyses ${ }^{122,123}$. Nominal significance level a was set to 0.05 . Biomarkers were categorized according to their functional pathways. To consider multiple testing, Bonferroniadjusted significance level $\alpha_{\text {adj }}$ was calculated within the following groups of biomarkers: inflammation, oxidative stress, endotoxemia and endothelial function $\left(\alpha_{\text {adj }}=\alpha / k\right.$, where $k$ is the number of biomarkers in each category: inflammation: $\mathrm{k}=9$; oxidative stress: $\mathrm{k}=6$; endotoxemia: $\mathrm{k}=2$; and endothelial function: $\mathrm{k}=3$ ). Analyses were performed using SAS software, version 9.4 (SAS Institute, Cary, NC, USA).

We examined the relationship between LBP (endotoxemia), inflammation and metabolic outcomes using structural equation models (SEM). A SEM estimates all model parameters simultaneously and thus assesses the strength of a particular relationship within the context of a model that can include both measured and constructed (latent) variables ${ }^{124}$. We hypothesized that LBP was associated with the cardiometabolic outcomes through full mediation by the latent (unobserved) variable inflammation inferred from the observed biomarkers CRP, TNF- $\alpha$ and IL-6. In the SEM, the estimation process aims to minimize the difference between the sampleand model-implied variance-covariance matrices. The latent variable was estimated by analysing the variance and covariance of the biomarkers (CRP, TNF- $\alpha$ and IL-6). The loading biomarker CRP was fixed to 1 and the residual covariances of the latent variables were set at zero. Two structural models were considered. In the first model, the studied cardiometabolic outcomes were obesity, dyslipidemia, insulin resistance and HTN. In the second model, MetS was the only outcome studied. In the two models, the outcome regressions were adjusted for CRT exposure, age at interview and sex. Figures 1 and 2 present the path diagrams corresponding to models 1 and 2, respectively. The conventional rules of SEM visualization were applied ${ }^{125}$. The diagonally weighted least squares (DWLS) estimator was used to handle the studied binary outcomes ${ }^{105}$. Standardized parameter estimates were presented in addition to their non standardized counterparts in order to compare the relative impact of variables measured on the different scales ${ }^{126}$. Model fit was evaluated using Comparative Fit Index (CFI), TLI 
(Tucker-Lewis index), Root Mean Square Error of Approximation (RMSEA), and Standardized Root Mean Square Residual (SRMR). The conventional cutoffs indicating a good model-data fit are CFI $>0.95$, TLI $>0.95$, RMSEA $<0.06$, and SRMR $<0.08^{127}$, but some recent studies have cautioned against their applying to ordered categorical data ${ }^{104,105}$.The SEM analyses were performed using R package Lavaan version 0.6-6.

\section{Data availability}

The data generated and analysed during the current study are not publicly available due to confidentiality reasons but are available from the corresponding author on reasonable request.

Received: 13 September 2018; Accepted: 12 October 2020

Published online: 09 December 2020

\section{References}

1. Baker, K. S. et al. Impact of treatment exposures on cardiovascular risk and insulin resistance in childhood cancer survivors. Cancer Epidemiol. Biomark. Prev. 22, 1954-1963. https://doi.org/10.1158/1055-9965.epi-13-0610 (2013).

2. Oeffinger, K. C. Are survivors of acute lymphoblastic leukemia (ALL) at increased risk of cardiovascular disease?. Pediatr. Blood Cancer 50, 462-467. https://doi.org/10.1002/pbc.21410 (2008) (discussion 468).

3. Nottage, K. A. et al. Metabolic syndrome and cardiovascular risk among long-term survivors of acute lymphoblastic leukaemia_From the St. Jude Lifetime Cohort. Br. J. Haematol. https://doi.org/10.1111/bjh.12754 (2014).

4. Levy, E. et al. Cardiometabolic risk factors in childhood, adolescent and young adult survivors of acute lymphoblastic leukemiaA petale cohort. Sci. Rep. 7, 17684. https://doi.org/10.1038/s41598-017-17716-0 (2017).

5. Withycombe, J. S. et al. Weight patterns in children with higher risk ALL: A report from the Children's Oncology Group (COG) for CCG 1961. Pediatr. Blood Cancer 53, 1249-1254. https://doi.org/10.1002/pbc.22237 (2009).

6. Morel, S., Delvin, E., Marcil, V. \& Levy, E. Intestinal dysbiosis and development of cardiometabolic disorders in childhood cancer survivors: acritical review. Antioxid. Redox Signal. https://doi.org/10.1089/ars.2020.8102 (2020).

7. Chua, L. L. et al. Reduced microbial diversity in adult survivors of childhood acute lymphoblastic leukemia and microbial associations with increased immune activation. Microbiome 5, 35. https://doi.org/10.1186/s40168-017-0250-1 (2017).

8. Ariffin, H. et al. Young adult survivors of childhood acute lymphoblastic leukemia show evidence of chronic inflammation and cellular aging. Cancer 123, 4207-4214. https://doi.org/10.1002/cncr.30857 (2017).

9. Azanan, M. S. et al. Immunity in young adult survivors of childhood leukemia is similar to the elderly rather than age-matched controls: Role of cytomegalovirus. Eur. J. Immunol. 46, 1715-1726. https://doi.org/10.1002/eji.201646356 (2016).

10. Lyon, D. E. et al. Relationship of systemic cytokine concentrations to cognitive function over two years in women with early stage breast cancer. J. Neuroimmunol. 301, 74-82. https://doi.org/10.1016/j.jneuroim.2016.11.002 (2016).

11. Cheung, Y. T. et al. Association of proinflammatory cytokines and chemotherapy-associated cognitive impairment in breast cancer patients: A multi-centered, prospective, cohort study. Ann. Oncol. 26, 1446-1451. https://doi.org/10.1093/annonc/mdv20 6 (2015).

12. Pusztai, L. et al. Changes in plasma levels of inflammatory cytokines in response to paclitaxel chemotherapy. Cytokine 25, 94-102 (2004).

13. Williams, A. M. et al. Associations between inflammatory markers and cognitive function in breast cancer patients receiving chemotherapy. J. Neuroimmunol. 314, 17-23. https://doi.org/10.1016/j.jneuroim.2017.10.005 (2018).

14. Ganz, P. A. et al. Does tumor necrosis factor-alpha (TNF-alpha) play a role in post-chemotherapy cerebral dysfunction?. Brain. Behav. Immun. 30(Suppl), S99-108. https://doi.org/10.1016/j.bbi.2012.07.015 (2013).

15. Pomykala, K. L. et al. The association between pro-inflammatory cytokines, regional cerebral metabolism, and cognitive complaints following adjuvant chemotherapy for breast cancer. Brain Imaging Behav. 7, 511-523. https://doi.org/10.1007/s1168 2-013-9243-2 (2013)

16. Kesler, S. et al. Reduced hippocampal volume and verbal memory performance associated with interleukin-6 and tumor necrosis factor-alpha levels in chemotherapy-treated breast cancer survivors. Brain Behav. Immun. 30(Suppl), S109-116. https://doi. org/10.1016/j.bbi.2012.05.017 (2013).

17. Bulow, B., Link, K., Ahren, B., Nilsson, A. S. \& Erfurth, E. M. Survivors of childhood acute lymphoblastic leukaemia, with radiation-induced GH deficiency, exhibit hyperleptinaemia and impaired insulin sensitivity, unaffected by 12 months of GH treatment. Clin. Endocrinol. 61, 683-691. https://doi.org/10.1111/j.1365-2265.2004.02149.x (2004).

18. Lee, S., Gungor, N., Bacha, F. \& Arslanian, S. Insulin resistance: Link to the components of the metabolic syndrome and biomarkers of endothelial dysfunction in youth. Diabetes Care 30, 2091-2097. https://doi.org/10.2337/dc07-0203 (2007).

19. Tonorezos, E. S. et al. Adipokines, body fatness, and insulin resistance among survivors of childhood leukemia. Pediatr. Blood Cancer 58, 31-36. https://doi.org/10.1002/pbc.22964 (2012).

20. Barbosa-Cortes, L. et al. Adipokines, insulin resistance, and adiposity as a predictors of metabolic syndrome in child survivors of lymphoma and acute lymphoblastic leukemia of a developing country. BMC Cancer 17, 125. https://doi.org/10.1186/s1288 5-017-3097-8 (2017).

21. Jahnukainen, K. et al. Increased body adiposity and serum leptin concentrations in very long-term adult male survivors of childhood acute lymphoblastic leukemia. Horm Res. Paediatr. 84, 108-115. https://doi.org/10.1159/000431092 (2015).

22. Kojima, C. et al. Adipocytokines in childhood cancer survivors and correlation with metabolic syndrome components. Pediatr. Int. 55, 438-442. https://doi.org/10.1111/ped.12087 (2013).

23. Jarvela, L. S. et al. Endothelial function in long-term survivors of childhood acute lymphoblastic leukemia: Effects of a homebased exercise program. Pediatr. Blood Cancer 60, 1546-1551. https://doi.org/10.1002/pbc.24565 (2013).

24. Chen, M. H., Colan, S. D. \& Diller, L. Cardiovascular disease: Cause of morbidity and mortality in adult survivors of childhood cancers. Circ. Res. 108, 619-628. https://doi.org/10.1161/CIRCRESAHA.110.224519 (2011).

25. Gurney, J. G. et al. Abdominal aortic calcification in young adult survivors of childhood acute lymphoblastic leukemia: Results from the St. Jude Lifetime Cohort study. Pediatr. Blood Cancer 59, 1307-1309. https://doi.org/10.1002/pbc.24134 (2012).

26. Mertens, A. C. et al. Cause-specific late mortality among 5-year survivors of childhood cancer: The Childhood Cancer Survivor Study. J. Natl. Cancer Inst. 100, 1368-1379. https://doi.org/10.1093/jnci/djn310 (2008).

27. Chao, C., Xu, L., Bell, E., Cooper, R. \& Mueller, L. Long-term health outcomes in survivors of childhood cancer diagnosed between 1990 and 2000 in a large US Integrated Health Care System. J. Pediatr. Hematol. Oncol. 38, 123-130. https://doi. org/10.1097/MPH.0000000000000492 (2016).

28. Chao, C. et al. Cardiovascular disease risk profiles in survivors of adolescent and young adult (AYA) cancer: The Kaiser Permanente AYA Cancer Survivors Study. J. Clin. Oncol. 34, 1626-1633. https://doi.org/10.1200/JCO.2015.65.5845 (2016).

29. Sadurska, E., Zaucha-Prazmo, A., Brodzisz, A., Kowalczyk, J. \& Ben-Skowronek, I. Premature atherosclerosis after treatment for acute lymphoblastic leukemia in childhood. Ann. Agric. Environ. Med. 25, 71-76. https://doi.org/10.5604/12321966.12306 80 (2018). 
30. Sulicka, J. et al. Elevated markers of inflammation and endothelial activation and increased counts of intermediate monocytes in adult survivors of childhood acute lymphoblastic leukemia. Immunobiology 218, 810-816. https://doi.org/10.1016/j.imbio 2012.09.003 (2013)

31. Masopustova, A. et al. Plethysmographic and biochemical markers in the diagnosis of endothelial dysfunction in pediatric acute lymphoblastic leukemia survivorsșnew applications. Physiol. Res. 67, 903-909. https://doi.org/10.33549/physiolres.93375 4 (2018).

32. de Sousa Almondes, K. G. et al. Selenium inadequacy is not associated with oxidative stress in child and adolescent acute lymphocytic leukemia survivors. Nutrition 30, 563-568 (2014).

33. Buttros, D. D. et al. Risk of metabolic syndrome in postmenopausal breast cancer survivors. Menopause 20, 448-454. https:// doi.org/10.1097/gme.0b013e318272bd4a (2013).

34. Conklin, K. A. Chemotherapy-associated oxidative stress: Impact on chemotherapeutic effectiveness. Integr. Cancer Ther. 3, 294-300. https://doi.org/10.1177/1534735404270335 (2004).

35. Irwin, M. E., Rivera-Del Valle, N. \& Chandra, J. Redox control of leukemia: From molecular mechanisms to therapeutic opportunities. Antioxid. Redox Signal 18, 1349-1383. https://doi.org/10.1089/ars.2011.4258 (2013).

36. Chandel, N. S. Mitochondria as signaling organelles. BMC Biol. 12, 34. https://doi.org/10.1186/1741-7007-12-34 (2014).

37. Lipshultz, S. E. et al. Impaired mitochondrial function is abrogated by dexrazoxane in doxorubicin-treated childhood acute lymphoblastic leukemia survivors. Cancer 122, 946-953. https://doi.org/10.1002/cncr.29872 (2016).

38. Wen, Q., Hu, Y., Ji, F. \& Qian, G. Mitochondrial DNA alterations of peripheral lymphocytes in acute lymphoblastic leukemia patients undergoing total body irradiation therapy. Radiat. Oncol. 6, 133. https://doi.org/10.1186/1748-717X-6-133 (2011).

39. Leahy, J. et al. Insight from mitochondrial functions and proteomics to understand cardiometabolic disorders in survivors of acute lymphoblastic leukemia. Metabolism https://doi.org/10.1016/j.metabol.2018.03.011 (2018).

40. Chassaing, B., Ley, R. E. \& Gewirtz, A. T. Intestinal epithelial cell toll-like receptor 5 regulates the intestinal microbiota to prevent low-grade inflammation and metabolic syndrome in mice. Gastroenterology https://doi.org/10.1053/j.gastro.2014.08.033 (2014).

41. Qin, J. et al. A metagenome-wide association study of gut microbiota in type 2 diabetes. Nature 490, 55-60. https://doi. org/10.1038/nature11450 (2012).

42. Le Chatelier, E. et al. Richness of human gut microbiome correlates with metabolic markers. Nature 500, 541-546. https://doi. org/10.1038/nature12506 (2013).

43. Wang, J. et al. Modulation of gut microbiota during probiotic-mediated attenuation of metabolic syndrome in high fat diet-fed mice. ISME J. https://doi.org/10.1038/ismej.2014.99 (2014).

44. Cani, P. D. et al. Changes in gut microbiota control metabolic endotoxemia-induced inflammation in high-fat diet-induced obesity and diabetes in mice. Diabetes 57, 1470-1481. https://doi.org/10.2337/db07-1403 (2008).

45. Karlsson, F. H. et al. Gut metagenome in European women with normal, impaired and diabetic glucose control. Nature 498, 99-103. https://doi.org/10.1038/nature12198 (2013).

46. Grube, B. J. et al. Lipopolysaccharide binding protein expression in primary human hepatocytes and HepG2 hepatoma cells. J. Biol. Chem. 269, 8477-8482 (1994).

47. Schroedl, W., Kleessen, B., Jaekel, L., Shehata, A. A. \& Krueger, M. Influence of the gut microbiota on blood acute-phase proteins. Scand. J. Immunol. 79, 299-304. https://doi.org/10.1111/sji.12161 (2014).

48. Parry, S. Fit Statistics Commonly Reported for CFA and SEM (Cornell Statistical Consulting Unit Cornell University, Ithaca, 2017).

49. Cangur, S. \& Ercan, I. Comparison of model fit indices used in structural equation modeling under multivariate normality. J. Mod. Appl. Stat. Methods 14, 14 (2015).

50. Lee, B. C. \& Lee, J. Cellular and molecular players in adipose tissue inflammation in the development of obesity-induced insulin resistance. Biochim. Biophys. Acta 446-462, 2014. https://doi.org/10.1016/j.bbadis.2013.05.017 (1842).

51. Srikanthan, K., Feyh, A., Visweshwar, H., Shapiro, J. I. \& Sodhi, K. Systematic review of metabolic syndrome biomarkers: A panel for early detection, management, and risk stratification in the west virginian population. Int. J. Med. Sci. 13, 25-38. https ://doi.org/10.7150/ijms.13800 (2016).

52. Reinehr, T., Stoffel-Wagner, B., Roth, C. L. \& Andler, W. High-sensitive C-reactive protein, tumor necrosis factor alpha, and cardiovascular risk factors before and after weight loss in obese children. Metabolism 54, 1155-1161. https://doi.org/10.1016/j. metabol.2005.03.022 (2005).

53. Berberoglu, M. Evaluation of the correlation between serum tumor necrosis factor-alpha and relative body mass index (RBMI) in childhood. J. Pediatr. Endocrinol. Metab. 14, 543-547 (2001).

54. Moon, Y. S., Kim, D. H. \& Song, D. K. Serum tumor necrosis factor-alpha levels and components of the metabolic syndrome in obese adolescents. Metabolism 53, 863-867 (2004).

55. Kolb-Bachofen, V. A review on the biological properties of C-reactive protein. Immunobiology 183, 133-145. https://doi. org/10.1016/S0171-2985(11)80193-2 (1991).

56. Pearson, T. A. et al. Markers of inflammation and cardiovascular disease: application to clinical and public health practice: A statement for healthcare professionals from the Centers for Disease Control and Prevention and the American Heart Association. Circulation 107, 499-511 (2003).

57. Giordano, P. et al. Endothelial dysfunction and cardiovascular risk factors in childhood acute lymphoblastic leukemia survivors. Int. J. Cardiol. 228, 621-627. https://doi.org/10.1016/j.ijcard.2016.11.025 (2017).

58. Latoch, E. et al. Adipokines and insulin resistance in young adult survivors of childhood cancer. Int. J. Endocrinol. 2016, 6349134. https://doi.org/10.1155/2016/6349134 (2016).

59. Esbenshade, A. J., Simmons, J. H., Koyama, T., Lindell, R. B. \& Friedman, D. L. Obesity and insulin resistance in pediatric acute lymphoblastic leukemia worsens during maintenance therapy. Pediatr. Blood Cancer 60, 1287-1291. https://doi.org/10.1002/ pbc.24489 (2013).

60. Beauloye, V., Steffens, M., Zech, F., Vermylen, C. \& Maiter, D. Characterization of insulin resistance in young adult survivors of childhood acute lymphoblastic leukaemia and non-Hodgkin lymphoma. Clin. Endocrinol. 78, 790-798. https://doi.org/10.1111/ cen.12047 (2013).

61. Medina-Bravo, P. et al. Decrease in serum adiponectin levels associated with visceral fat accumulation independent of pubertal stage in children and adolescents. Arch. Med. Res. 42, 115-121. https://doi.org/10.1016/j.arcmed.2011.03.002 (2011).

62. Asayama, K. et al. Decrease in serum adiponectin level due to obesity and visceral fat accumulation in children. Obes. Res. 11, 1072-1079. https://doi.org/10.1038/oby.2003.147 (2003).

63. Sanip, Z., Ariffin, F. D., Al-Tahami, B. A., Sulaiman, W. A. \& Rasool, A. H. Obesity indices and metabolic markers are related to hs-CRP and adiponectin levels in overweight and obese females. Obes. Res. Clin. Pract. 7, e315-320. https://doi.org/10.1016/j. orcp.2012.05.002 (2013).

64. Marsche, G. et al. Adiponectin predicts high-density lipoprotein cholesterol efflux capacity in adults irrespective of body mass index and fat distribution. J. Clin. Endocrinol. Metab. 102, 4117-4123. https://doi.org/10.1210/jc.2017-00933 (2017).

65. Schneider, J. G., von Eynatten, M., Schiekofer, S., Nawroth, P. P. \& Dugi, K. A. Low plasma adiponectin levels are associated with increased hepatic lipase activity in vivo. Diabetes Care 28, 2181-2186 (2005).

66. Follin, C. et al. Associations between metabolic risk factors and the hypothalamic volume in childhood leukemia survivors treated with cranial radiotherapy. PLoS ONE 11, e0147575. https://doi.org/10.1371/journal.pone.0147575 (2016). 
67. Saddi-Rosa, P., Oliveira, C. S., Giuffrida, F. M. \& Reis, A. F. Visfatin, glucose metabolism and vascular disease: A review of evidence. Diabetol. Metab. Syndr. 2, 21. https://doi.org/10.1186/1758-5996-2-21 (2010).

68. Fukuhara, A. et al. Visfatin: A protein secreted by visceral fat that mimics the effects of insulin. Science 307, 426-430. https:// doi.org/10.1126/science.1097243 (2005).

69. Qin, S. et al. Effects of visfatin gene overexpression on insulin sensitivity in the insulin-resistant rats induced by high-fat diet. Zhonghua Yi Xue Za Zhi 88, 365-368 (2008).

70. Sun, Q. et al. Overexpression of visfatin/PBEF/Nampt alters whole-body insulin sensitivity and lipid profile in rats. Ann. Med. 41, 311-320. https://doi.org/10.1080/07853890902729760 (2009).

71. Revollo, J. R. et al. Nampt/PBEF/Visfatin regulates insulin secretion in beta cells as a systemic NAD biosynthetic enzyme. Cell Metab. 6, 363-375. https://doi.org/10.1016/j.cmet.2007.09.003 (2007).

72. Skoczen, S. et al. Visfatin concentrations in children with leukemia before and after stem cell transplantation. Exp. Hematol. 42, 252-260. https://doi.org/10.1016/j.exphem.2013.12.006 (2014).

73. Siviero-Miachon, A. A. et al. Visfatin is a positive predictor of bone mineral density in young survivors of acute lymphocytic leukemia. J. Bone Miner. Metab. 35, 73-82. https://doi.org/10.1007/s00774-015-0728-5 (2017).

74. Brouwer, C. A. et al. Endothelial damage in long-term survivors of childhood cancer. J. Clin. Oncol. 31, 3906-3913. https://doi. org/10.1200/JCO.2012.46.6086 (2013).

75. Sun, L. et al. A marker of endotoxemia is associated with obesity and related metabolic disorders in apparently healthy Chinese. Diabetes Care 33, 1925-1932. https://doi.org/10.2337/dc10-0340 (2010).

76. Troseid, M. et al. Plasma lipopolysaccharide is closely associated with glycemic control and abdominal obesity: Evidence from bariatric surgery. Diabetes Care 36, 3627-3632. https://doi.org/10.2337/dc13-0451 (2013).

77. Liang, H., Hussey, S. E., Sanchez-Avila, A., Tantiwong, P. \& Musi, N. Effect of lipopolysaccharide on inflammation and insulin action in human muscle. PLoS ONE 8, e63983. https://doi.org/10.1371/journal.pone.0063983 (2013).

78. Jayashree, B. et al. Increased circulatory levels of lipopolysaccharide (LPS) and zonulin signify novel biomarkers of proinflammation in patients with type 2 diabetes. Mol. Cell Biochem. 388, 203-210. https://doi.org/10.1007/s11010-013-1911-4 (2014).

79. Creely, S. J. et al. Lipopolysaccharide activates an innate immune system response in human adipose tissue in obesity and type 2 diabetes. Am. J. Physiol. Endocrinol. Metab. 292, E740-747. https://doi.org/10.1152/ajpendo.00302.2006 (2007).

80. Munford, R. S. Detoxifying endotoxin: time, place and person. J. Endotoxin Res. 11, 69-84. https://doi.org/10.1179/096805105X 35161 (2005).

81. Laugerette, F. et al. Oil composition of high-fat diet affects metabolic inflammation differently in connection with endotoxin receptors in mice. Am. J. Physiol. Endocrinol. Metab. 302, E374-386. https://doi.org/10.1152/ajpendo.00314.2011 (2012).

82. Citronberg, J. S. et al. Reliability of plasma lipopolysaccharide-binding protein (LBP) from repeated measures in healthy adults. Cancer Causes Control 27, 1163-1166. https://doi.org/10.1007/s10552-016-0783-9 (2016).

83. Lu, Y. C., Yeh, W. C. \& Ohashi, P. S. LPS/TLR4 signal transduction pathway. Cytokine 42, 145-151. https://doi.org/10.1016/j. cyto.2008.01.006 (2008).

84. Kim, J. J. \& Sears, D. D. TLR4 and insulin resistance. Gastroenterol. Res. Pract. https://doi.org/10.1155/2010/212563 (2010).

85. Wurfel, M. M., Hailman, E. \& Wright, S. D. Soluble CD14 acts as a shuttle in the neutralization of lipopolysaccharide (LPS) by LPS-binding protein and reconstituted high density lipoprotein. J. Exp. Med. 181, 1743-1754 (1995).

86. Gonzalez-Quintela, A. et al. Determinants of serum concentrations of lipopolysaccharide-binding protein (LBP) in the adult population: The role of obesity. PLoS ONE 8, e54600. https://doi.org/10.1371/journal.pone.0054600 (2013).

87. Lassenius, M. I. et al. Bacterial endotoxin activity in human serum is associated with dyslipidemia, insulin resistance, obesity, and chronic inflammation. Diabetes Care 34, 1809-1815. https://doi.org/10.2337/dc10-2197 (2011).

88. Saghafi-Asl, M., Amiri, P., Naghizadeh, M., Ghavami, S. M. \& Karamzad, N. Association of endotoxaemia with serum free fatty acids in metabolically healthy and unhealthy abdominally obese individuals: A case-control study in northwest of Iran. BMJ Open 7, e015910. https://doi.org/10.1136/bmjopen-2017-015910 (2017).

89. Liu, X. et al. Lipopolysaccharide binding protein, obesity status and incidence of metabolic syndrome: A prospective study among middle-aged and older Chinese. Diabetologia 57, 1834-1841. https://doi.org/10.1007/s00125-014-3288-7 (2014).

90. Tilves, C. M. et al. Association of lipopolysaccharide-binding protein with aging-related adiposity change and prediabetes among African ancestry men. Diabetes Care 39, 385-391. https://doi.org/10.2337/dc15-1777 (2016).

91. Kim, K. E. et al. Lipopolysaccharide-binding protein plasma levels as a biomarker of obesity-related insulin resistance in adolescents. Korean J. Pediatr. 59, 231-238. https://doi.org/10.3345/kjp.2016.59.5.231 (2016).

92. Moreno-Navarrete, J. M. et al. Circulating lipopolysaccharide-binding protein (LBP) as a marker of obesity-related insulin resistance. Int. J. Obes. 36, 1442-1449. https://doi.org/10.1038/ijo.2011.256 (2012).

93. Holvoet, P., Lee, D. H., Steffes, M., Gross, M. \& Jacobs, D. R. Jr. Association between circulating oxidized low-density lipoprotein and incidence of the metabolic syndrome. JAMA 299, 2287-2293. https://doi.org/10.1001/jama.299.19.2287 (2008).

94. Trpkovic, A. et al. Oxidized low-density lipoprotein as a biomarker of cardiovascular diseases. Crit. Rev. Clin. Lab. Sci. 52, 70-85. https://doi.org/10.3109/10408363.2014.992063 (2015).

95. Kilic, I. D. et al. Circulating adhesion molecules and arterial stiffness. Cardiovasc. J. Afr. 26, 21-24. https://doi.org/10.5830/ CVJA-2014-060 (2015).

96. Luc, G. et al. Circulating soluble adhesion molecules ICAM-1 and VCAM-1 and incident coronary heart disease: The PRIME Study. Atherosclerosis 170, 169-176 (2003).

97. Malik, I. et al. Soluble adhesion molecules and prediction of coronary heart disease: A prospective study and meta-analysis. Lancet 358, 971-976. https://doi.org/10.1016/S0140-6736(01)06104-9 (2001).

98. Shai, I. et al. Soluble intercellular adhesion molecules, soluble vascular cell adhesion molecules, and risk of coronary heart disease. Obesity 14, 2099-2106. https://doi.org/10.1038/oby.2006.245 (2006).

99. Pontiroli, A. E. et al. Body weight and glucose metabolism have a different effect on circulating levels of ICAM-1, E-selectin, and endothelin-1 in humans. Eur. J. Endocrinol. 150, 195-200 (2004).

100. Zanni, M. V., Stanley, T. L., Makimura, H., Chen, C. Y. \& Grinspoon, S. K. Effects of TNF-alpha antagonism on E-selectin in obese subjects with metabolic dysregulation. Clin. Endocrinol. 73, 48-54. https://doi.org/10.1111/j.1365-2265.2009.03741.x (2010).

101. Meigs, J. B., Hu, F. B., Rifai, N. \& Manson, J. E. Biomarkers of endothelial dysfunction and risk of type 2 diabetes mellitus. JAMA 291, 1978-1986. https://doi.org/10.1001/jama.291.16.1978 (2004).

102. Stepanikova, I., Oates, G. R. \& Bateman, L. B. Does one size fit all? The role of body mass index and waist circumference in systemic inflammation in midlife by race and gender. Ethn. Health 22, 169-183. https://doi.org/10.1080/13557858.2016.12356 81 (2017).

103. Wolf, E. J., Harrington, K. M., Clark, S. L. \& Miller, M. W. Sample size requirements for structural equation models: An evaluation of power, bias, and solution propriety. Educ. Psychol. Meas. 76, 913-934. https://doi.org/10.1177/0013164413495237 (2013).

104. Xia, Y. \& Yang, Y. RMSEA, CFI, and TLI in structural equation modeling with ordered categorical data: The story they tell depends on the estimation methods. Behav. Res. Methods 51, 409-428 (2019).

105. Xia, Y. \& Yang, Y. The influence of number of categories and threshold values on fit indices in structural equation modeling with ordered categorical data. Multivar. Behav. Res. 53, 731-755 (2018). 
106. Marcoux, S. et al. The PETALE study: Late adverse effects and biomarkers in childhood acute lymphoblastic leukemia survivors. Pediatr. Blood Cancer https://doi.org/10.1002/pbc.26361 (2017).

107. Vrooman, L. M. et al. The low incidence of secondary acute myelogenous leukaemia in children and adolescents treated with dexrazoxane for acute lymphoblastic leukaemia: A report from the Dana-Farber Cancer Institute ALL Consortium. Eur. J. Cancer 47, 1373-1379. https://doi.org/10.1016/j.ejca.2011.03.022 (2011).

108. Van den Broeck, J., Willie, D. \& Younger, N. The World Health Organization child growth standards: Expected implications for clinical and epidemiological research. Eur. J. Pediatr. 168, 247-251. https://doi.org/10.1007/s00431-008-0796-9 (2009).

109. Alberti, K. G., Zimmet, P., Shaw, J. \& Group, I. D. F. E. T. F. C. The metabolic syndrome-A new worldwide definition. Lancet 366, 1059-1062. https://doi.org/10.1016/S0140-6736(05)67402-8 (2005).

110. National Cholesterol Education Program Expert Panel on Detection, E. \& Treatment of High Blood Cholesterol in, A. Third Report of the National Cholesterol Education Program (NCEP) expert panel on detection, evaluation, and treatment of high blood cholesterol in adults (adult treatment panel III) final report. Circulation 106, 3143-3421 (2002).

111. Katzmarzyk, P. T. Waist circumference percentiles for Canadian youth 11-18y of age. Eur. J. Clin. Nutr. 58, 1011-1015. https:// doi.org/10.1038/sj.ejcn.1601924 (2004).

112. Canadian Diabetes Association Clinical Practice Guidelines Expert, C, Goldenberg, R. \& Punthakee, Z. Definition, classification and diagnosis of diabetes, prediabetes and metabolic syndrome. Can. J. Diabetes 37(Suppl 1), S8-11. https://doi.org/10.1016/j. jcjd.2013.01.011 (2013).

113. Allard, P. et al. Distribution of fasting plasma insulin, free fatty acids, and glucose concentrations and of homeostasis model assessment of insulin resistance in a representative sample of Quebec children and adolescents. Clin. Chem. 49, 644-649 (2003).

114. Stern, R. H. The new hypertension guidelines. J. Clin. Hypertens. 15, 748-751. https://doi.org/10.1111/jch.12171 (2013).

115. Paradis, G., Tremblay, M. S., Janssen, I., Chiolero, A. \& Bushnik, T. Blood pressure in Canadian children and adolescents. Health Rep. 21, 15-22 (2010).

116. Genest, J. et al. 2009 Canadian Cardiovascular Society/Canadian guidelines for the diagnosis and treatment of dyslipidemia and prevention of cardiovascular disease in the adult - 2009 recommendations. Can. J. Cardiol. 25, 567-579 (2009).

117. FOR, E. P. O. I. G. \& CHILDREN, R. R. I. Expert panel on integrated guidelines for cardiovascular health and risk reduction in children and adolescents: Summary report. Pediatrics 128, S213 (2011).

118. Zimmet, P. et al. The metabolic syndrome in children and adolescents - an IDF consensus report. Pediatr. Diabetes 8, 299-306. https://doi.org/10.1111/j.1399-5448.2007.00271.x (2007).

119. Griffith, O. W. Determination of glutathione and glutathione disulfide using glutathione reductase and 2-vinylpyridine. Anal. Biochem. 106, 207-212 (1980).

120. Lavoie, J. C. \& Chessex, P. Gender and maturation affect glutathione status in human neonatal tissues. Free Radic. Biol. Med. 23, 648-657 (1997).

121. Livak, K. J. \& Schmittgen, T. D. Analysis of relative gene expression data using real-time quantitative PCR and the 2(-Delta Delta C(T)) method. Methods 25, 402-408. https://doi.org/10.1006/meth.2001.1262 (2001).

122. Allison, P. D. Logistic Regression Using SAS": Theory and Application: Theory and Application 2nd edn. (SAS Institute, Cary, 2012).

123. 123Allison, P. D. In SAS Global Forum. 1-11.

124. Dion, P. A. Interpreting structural equation modeling results: A reply to Martin and Cullen. J. Bus. Ethics 83, 365-368 (2008).

125. Epskamp, S. semPlot: Unified visualizations of structural equation models. Struct. Equ. Model. Multidiscip. J. 22, 474-483. https ://doi.org/10.1080/10705511.2014.937847 (2015).

126. Kwan, J. L. Y. \& Chan, W. Comparing standardized coefficients in structural equation modeling: A model reparameterization approach. Behav. Res. Methods 43, 730-745. https://doi.org/10.3758/s13428-011-0088-6 (2011).

127. Hu, L. T. \& Bentler, P. M. Cutoff criteria for fit indexes in covariance structure analysis: Conventional criteria versus new alternatives. Struct. Equ. Model. Multidiscip. J. 6, 1-55 (1999).

\section{Acknowledgements}

This work was supported by the Institute of Cancer Research (ICR) of the Canadian Institutes of Health Research (CIHR), in collaboration with C17 Council, Canadian Cancer Society (CCS), Cancer Research Society (CRS), Garron Family Cancer Centre at the Hospital for Sick Children, Ontario Institute for Cancer Research (OICR) and Pediatric Oncology Group of Ontario (POGO) (grant number: TCF 118694). SM is holding a fellowship from the Fonds de la Recherche du Québec-Santé (FRQ-S); PL is holding a fellowship from the Fonds de la Recherche du Québec-Santé (FRQ-S); VT received a Canderel fellowship from the Institut du cancer de Montréal (ICM); FR holds a FRQ-S junior II career awards (33070) and received support from the ICM for this project; GL is a Research Scholar of the Fonds de recherche Québec-Santé; VM was funded by a Transition grant from the Cole Foundation. The sponsors had no involvement in study design; in the collection, analysis and interpretation of data; in the writing of the report; and in the decision to submit the article for publication.

\section{Author contributions}

D.S., M.K., E.L., S.D., S.L., C.L. and V.M. conceived the study and participated in the design and coordination. V.M. and S.M. collected the cardiometabolic data. V.M., J.E. and S.M. classified participants according to their metabolic status. A.F. and S.M. performed the biomarkers analysis at SJUHC. N.M., V.T., G.B.C. and F.R. performed circulating biomarkers analysis using V-PLEX assays at the CRCHUM. S.M., P.L., J.E., S.D. and V.M. analyzed data. P.L., M.S., S.M., V.M., S.D. and G.L. planned the statistical analysis. P.L., M.S. and S.M. performed the statistical analysis. S.M., P.L. and V.M. interpreted the data. S.M., P.L., A.F., M.S., S.D., E.L., D.S. and V.M. contributed to the writing of the manuscript. All authors have read and approved this manuscript.

\section{Competing interests}

The authors declare no competing interests.

\section{Additional information}

Supplementary Information The online version contains supplementary material available at https://doi. org/10.1038/s41598-020-78493-X.

Correspondence and requests for materials should be addressed to V.M.

Reprints and permissions information is available at www.nature.com/reprints. 
Publisher's note Springer Nature remains neutral with regard to jurisdictional claims in published maps and institutional affiliations.

(c) (1) Open Access This article is licensed under a Creative Commons Attribution 4.0 International License, which permits use, sharing, adaptation, distribution and reproduction in any medium or format, as long as you give appropriate credit to the original author(s) and the source, provide a link to the Creative Commons licence, and indicate if changes were made. The images or other third party material in this article are included in the article's Creative Commons licence, unless indicated otherwise in a credit line to the material. If material is not included in the article's Creative Commons licence and your intended use is not permitted by statutory regulation or exceeds the permitted use, you will need to obtain permission directly from the copyright holder. To view a copy of this licence, visit http://creativecommons.org/licenses/by/4.0/.

(c) The Author(s) 2020 